\title{
What we count dictates how we count: A tale of two encodings
}

\author{
Hippolyte Gros ${ }^{1} *$ • Jean-Pierre Thibaut ${ }^{2}$ Emmanuel Sander ${ }^{1}$
}

${ }^{1}$ University of Geneva. ${ }^{2}$ University of Bourgogne Franche-Comté

\begin{abstract}
:
We argue that what we count has a crucial impact on how we count, to the extent that even adults may have difficulty using elementary mathematical notions in concrete situations. Specifically, we investigate how the use of certain types of quantities (durations, heights, number of floors) may emphasize the ordinality of the numbers featured in a problem, whereas other quantities (collections, weights, prices) may emphasize the cardinality of the depicted numerical situations. We suggest that this distinction leads to the construction of one of two possible encodings, either a cardinal or an ordinal representation. This difference should, in turn, constrain the way we approach problems, influencing our mathematical reasoning in multiple activities. This hypothesis is tested in six experiments $(N=$ 916), using different versions of multiple-strategy arithmetic word problems. We show that the distinction between cardinal and ordinal quantities predicts problem sorting (Experiment 1), perception of similarity between problems (Experiment 2), direct problem comparison (Experiment 3), choice of a solving algorithm (Experiment 4), problem solvability estimation (Experiment 5) and solution validity assessment (Experiment 6). The results provide converging clues shedding light into the fundamental importance of the cardinal versus ordinal distinction on adults' reasoning about numerical situations. Overall, we report multiple evidence that general, non-mathematical knowledge associated with the use of different quantities shapes adults' encoding, recoding and solving of mathematical word problems. The implications regarding mathematical cognition and theories of arithmetic problem solving are discussed.
\end{abstract}

Graphical abstract:

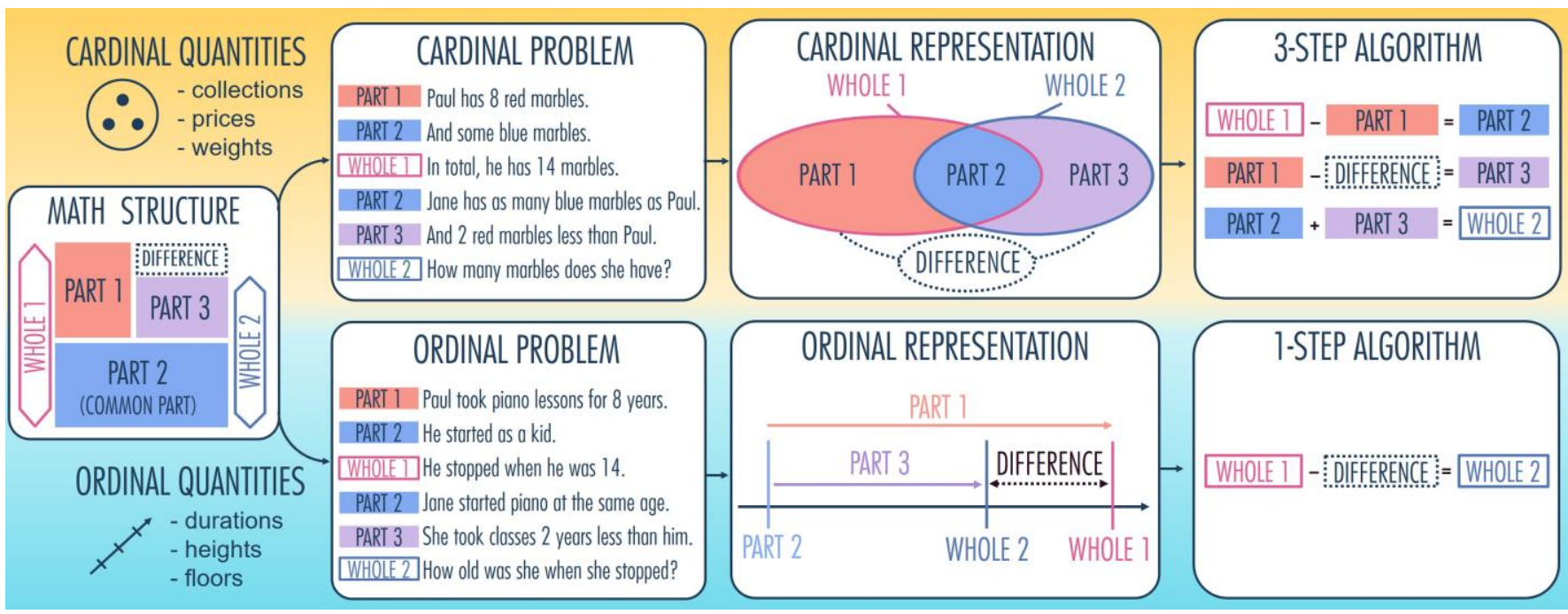

Keywords: arithmetic problem solving; cardinality; mathematical reasoning; mental model; numerical cognition; ordinality. 


\section{Introduction}

Mathematical notions are frequently used in everyday life (Northcote \& Marshall, 2016). Whether someone is calculating a bus fare, estimating the duration of the ride, deciding whether to go up the stairs or take the elevator, assessing if the added weight of everyone in the elevator is below the weight limit, counting how many cookies to buy for the coworkers, or evaluating at which height to put the cookie jar so that the kids won't reach it, people use mathematics in many aspects of their lives. However, being able to perform those quick calculations on a daily basis requires the ability to use mathematical notions in very tangible situations (Schoenfeld, 2009). Mastering such a skill is the focus of a long learning process taking place in school, notably involving mathematical word problems (Daroczy, Wolska, Meurers, \& Nuerk, 2015; Stacey, 2005). But while using arithmetic notions in daily-life might seem effortless to most adults, we argue that some underlying pervasive influence remains: in fact, even when using the most elementary mathematical notions, we have reasons to believe that what we count has a deep impact on how we count. This entails that even adults may be significantly better at using certain fundamental mathematical notions in situations involving bus rides, elevator trips or cookie jar heights than in situations pertaining to bus ticket prices, elevator weight limits or cookie counting. In this paper, we argue that such concrete situations evoke one of two distinct conceptions of numerical situations; they either emphasize the cardinal property of numbers or their ordinal property (see below). This difference leads to one of two possible encodings, which in turn constrain the way we represent word problems, influencing our mathematical reasoning in multiple activities.

\section{An ontological distinction between cardinal and ordinal situations}

This distinction between cardinality and ordinality is fundamental in mathematics, especially in set theory (Dantzig, 1945; Frege, 1980; Russell, 1919; Simon, 1997; Suppes, 1972). In common usage, ordinal numbers describe the numerical position of an object in an ordered sequence (i.e. $1^{\text {st }}, 2^{\text {nd }}, 3^{\text {rd }}$, etc.), whereas cardinal numbers refer to the general concept of quantity by designating the total number of entities within a set (Fuson, 1988; Wasner, Moeller, Fischer, \& Nuerk, 2015). But this distinction has far-reaching implications beyond set theory and mathematics and, we believe, influences the very way humans comprehend numerosity in the world.

According to Piaget (1941/1965), the relationship between cardinality and ordinality is central to the notion of number (Fuson, 1988). Indeed, the two ideas are necessarily intertwined, since the cardinal and ordinal meanings of numbers are two sides of the same coin, two properties intrinsically tied to our use of numbers. As Fuson (1988, p. 363) puts it, "any ordinal number refers to the particular entity within a linear ordering that is 
preceded by a cardinal number one less than it". However, this interdependency between ordinality and cardinality does not preclude that a crucial difference between conceiving of numbers as cardinal count values or as order labels could exist.

From a developmental perspective, the seminal work of Gelman and Gallistel (1978) on counting principles introduced the idea that the understanding of the cardinal and ordinal properties of numbers could develop separately in children. In their work, Gelman and Gallistel notably argued that children needed to learn the "stableorder-principle" and the "cardinal principle" in order to become proficient counters. Mastery of the stable-orderprinciple means that a child has learned that the list of words used to count must be used in a definite and repeatable order; it can be linked to the development of the notion of ordinality. The cardinal-principle, on the other hand, refers to the understanding that the number name allocated to the final entity in a collection corresponds to the total number of entities being counted. Ever since this work, investigations have been conducted to study how children learn to master both properties of numbers.

Children's ability to comprehend and use the notion of cardinality in counting was thus scrutinized by numerous studies who showed its slow and sequential development over the first years of life (e.g. Bermejo, 1996; Condry \& Spelke, 2008; Le Corre \& Carey, 2007; Sarnecka \& Lee, 2009; Wynn, 1992). As for the development of the ordinal meaning of numbers, it has been suggested that children do not learn to use ordinal labels such as "first" and "second" before they are 4 or 5 years old (Fischer \& Beckey, 1990; Miller, Major, Shu, \& Zhang, 2000; Miller, Marcovitch, Boseovski, \& Lewkowicz, 2015). In recent years, the comparison of the developmental trajectories of the sense of cardinality and the sense of ordinality has received increasing research interest, with converging results showing that children tend to use cardinality before succeeding in ordinality tasks (Colomé \& Noël, 2012; Meyer, Barbiers, \& Weerman, 2016; Wasner et al., 2015). Further evidence for the importance of this distinction can be found in the brain; Delazer and Butterworth (1997) reported that a patient who suffered a cerebral lesion was left with impaired access to the cardinal meaning of numbers, but a selectively preserved access to their ordinal meaning. On the other hand, Turconi and Seron (2002) reported that a patient suffering from lesions in the right parietal occipital junction and in the left posterior parietal cortex struggled to judge ordinal relationships between numbers whereas he remained able to understand cardinality to a certain extent, as shown by his successful completion of number comparison tasks. A similar dissociation was found by Chen, $\mathrm{Xu}$, Shang, Peng, and Luo (2014) who reported that lesions in the left parietal lobe resulted in a selective impairment of order meaning among one of their patients. This dissociation between the two meanings of numbers was further supported by EEG studies who found that event-related potentials differed both in terms of timing and topography 
between order and quantity tasks (Rubinsten, Dana, Lavro, \& Berger, 2013; Turconi, Jemel, Rossion, \& Seron, 2004). Furthermore, an fMRI study by Lyons and Beilock (2013) showed that while cardinal and ordinal judgments tasks involved a similar frontoparietal network for non-symbolic quantities (i.e. dot arrays), the use of symbolic numbers (i.e. numerals) led to the activation of different networks for cardinal and ordinal tasks. Similarly, Matejko, Hutchison, and Ansari (2019) found evidence of ordinal processing in the left inferior parietal cortex of adults, while no such evidence was found for cardinal processing.

In recent years, behavioral studies have used distinct ordinality and cardinality tasks to investigate the orthogonality of these two sides of number processing. Using digit ordering and number comparison tasks, they showed that basic numerical measures of ordinal and cardinal processing differentially predict arithmetic performance and math achievement scores (Goffin \& Ansari, 2016; Sasanguie, Lyons, De Smedt, \& Reynvoet, 2017). Overall, these studies show the importance of the distinction between cardinality and ordinality in numerical cognition. Processing the cardinality and ordinality of numerical symbols involves distinct neural underpinnings, each equally important and each predicting independent variance in arithmetic and mathematical proficiency. As Lyons, Vogel, and Ansari put it (2016), while research has initially focused on cardinal processing, "overlooking the contribution of ordinality to how we process numbers is likely a major oversight" (p. 206).

Despite this growing body of research on cardinal and ordinal tasks, little is known about adults' ability to perceive either the cardinality or the ordinality of complex numerical situations evoking those two sides of numbers. Can situations sharing the same mathematical deep structure highlight either cardinality or ordinality, depending on the daily-life knowledge they are imbued with? This paper aims at answering this crucial issue, by focusing on adults' ability to use basic mathematical notions in situations meant to emphasize either the cardinal or the ordinal aspect of numbers. Indeed, we propose to investigate whether the presence of different quantities (e.g. weights versus durations) in the wording of otherwise mathematically identical problems may lead even adults to build either a cardinal or an ordinal encoding of the depicted situations. We thus scrutinize the claim that this distinction will, in turn, lead to clear-cut differences in the way these problems are categorized, compared, and solved.

\section{The role of semantic knowledge in problem representation}

Our main hypothesis is that the distinction between cardinal quantities and ordinal quantities will lead to clearcut differences in the way arithmetic word problems are categorized, compared, and solved. This claim builds upon the literature on content effects in arithmetic word problems, which suggests that mathematically irrelevant semantic information may induce specific problem representations and that may interfere with arithmetic word 
problem solving. In 1995, Bassok, Wu, and Olseth showed that semantic knowledge linked to the different entities featured in a problem statement influenced analogical transfer between problems. They taught participants the algorithmic solution of a problem whose cover story depicted either objects assigned to people (e.g. computers given to secretaries) or people assigned to other people (e.g. doctors from one hospital assigned to doctors from another hospital). They then evaluated how well participants performed on problems sharing the same solution principle. Depending on whether the entities in the transfer problem had typically symmetrical roles (people and people) or typically asymmetrical roles (objects and people), participants' performance varied. Those results suggested that variations of semantic knowledge lead to different representations being abstracted. These "interpreted structures" either facilitated or hindered transfer depending on how well they mapped onto the problems' mathematical structure. Similarly, Bassok, Chase, and Martin (1998) asked participants to create addition or division word problems using specific sets of entities, either linked by a functional semantic relation (e.g. the container/content relation between fruit baskets and oranges) or by a collateral relation (e.g. oranges and apples belonging to the same superordinate "fruit" category). They showed that participants tended to propose division problems when the semantic knowledge induced by the entities evoked a functional relation, whereas they created addition problems when the entities were different kinds of fruits or other collateral elements.

Thus, the semantic knowledge induced by the entities described in the problem statement seems to influence the representation that participants encode. Bassok (2001) theorized this process by stating that an interpreted mathematical structure is abstracted by the participants, based on the semantic relations depicted in the problem statements (e.g. container/content or assigned/receiver relations). This interpreted structure can be semantically aligned or misaligned with the objective mathematical structure of the problem, depending on whether the two structures can be mapped onto each other (Bassok, 2001). For instance, the interpreted structure of a problem involving oranges and baskets will be semantically aligned with division, whereas the interpreted structure of a problem involving oranges and apples will be semantically aligned with division instead. In support of this view, Bassok, Pedigo and Oskarsson's work (2008) on the priming of addition facts by different pairs of words is enlightening. They showed that categorically related words (e.g. tigers and cheetahs) prime addition facts, whereas unrelated words (e.g. lungs and statues), as well as functionally related words (e.g. bears and claws), do not exert such priming. In other words, pairs of words semantically aligned with addition elicit an automatic retrieval of addition facts, whereas words misaligned with the structure of addition do not. Taken together, those studies suggest that the semantics induced by the entities manipulated in the problem statements influence the representational processes at play: non-mathematical properties of said entities constrain the operations selected 
by the participants.

\section{Preliminary work on arithmetic word problem representations}

In this paper, we push this point several steps further in the context of the cardinal-ordinal distinction. We investigate, with adults, the claim that the semantic knowledge about the entities described in a problem can evoke either an ordinal or a cardinal representation of the described situation, subsequently shaping mathematical reasoning. This idea comes from preliminary work on the perception of cardinality and ordinality in arithmetic word problems, undertaken by Gamo, Sander, and Richard (2010) in a study with $4^{\text {th }}$ and $5^{\text {th }}$ graders. The authors created multiple-solution word problems sharing the same mathematical structure but differing in the type of quantities they used. Their problem statements revolved around, respectively, the number of family members in a hotel (family problems), the price of a series of items (price problems) or the age of a protagonist (age problems) (see Table 1). Gamo et al. hypothesized that family problems and price problems would both lead to a cardinal encoding of the situation described, whereas age problems would lead to an ordinal encoding of the situation. Consider the family problem (Table 1, left column). Gamo et al. showed that most participants solved it using a 3-step algorithm: $9-5=4 ; 5-3=2 ; 4+2=6$. Note, however, that this problem can also be solved with a onestep algorithm: $9-3=6$. Using this algorithm requires the participant to realize that since the Roberts are present at the hotel during both vacations, then the difference between the number of people in the Richards' and in the Dumas' families is equal to the difference between the total number of people at the hotel. Thus, calculating the number of people in the Roberts' family $(9-5=4)$ or the number of people in the Dumas' family $(5-3=2)$ is not necessary to find the solution.

Table 1: Family, price and age problems used in Gamo et al. (2010).

\begin{tabular}{|c|c|c|}
\hline Family problem & Price problem & Age problem \\
\hline $\begin{array}{l}\text { In the Richards' family, there are } 5 \\
\text { persons. When the Richards go on } \\
\text { vacation with the Roberts, there are } 9 \\
\text { persons at the hotel. In the Dumas' } \\
\text { family, there are } 3 \text { people less than in } \\
\text { the Richards' family. The Roberts go } \\
\text { on vacation with the Dumas. How }\end{array}$ & $\begin{array}{l}\text { John bought an } 8 \text {-Euro exercise book } \\
\text { and a pair of scissors. He paid } 14 \\
\text { Euros. A pen costs } 3 \text { Euros less than } \\
\text { the exercise book. Paul bought scissors } \\
\text { and a pen. How much did he pay? }\end{array}$ & $\begin{array}{l}\text { Antoine attended painting classes at } \\
\text { the art school for } 8 \text { years and stopped } \\
\text { when he was } 17 \text { years old. Jean began } \\
\text { at the same age as Antoine and } \\
\text { attended the course for } 2 \text { years less. At } \\
\text { what age did Jean stop attending the } \\
\text { classes? }\end{array}$ \\
\hline
\end{tabular}

Similarly, most participants use a 3 -step algorithm to solve the price problem (Table 1, middle column). This algorithm consists in calculating the price of the pen and the price of the scissors, and then adding them up: $14-$ $8=6 ; 8-3=5 ; 6+5=11$. But a 1-step algorithm can also be used to solve this problem: $14-3=11$. However, only participants who notice that John and Paul both bought scissors may also understand that the difference between the price of the exercise book and the price of the pen is equal to the difference between what John and 
Paul paid in total, and thus be able to find this 1-step algorithm. Lastly, the age problem (Table 1, right column) could also be solved using a 3 -step algorithm $(17-8=9 ; 8-2=6 ; 9+6=15)$ or a 1 -step algorithm $(17-2=$ 15). Gamo et al. (2010) found that participants used the 1-step algorithm more often on such problems, even though all three problems were isomorphic and shared the exact same mathematical structure (see Fig. 1).

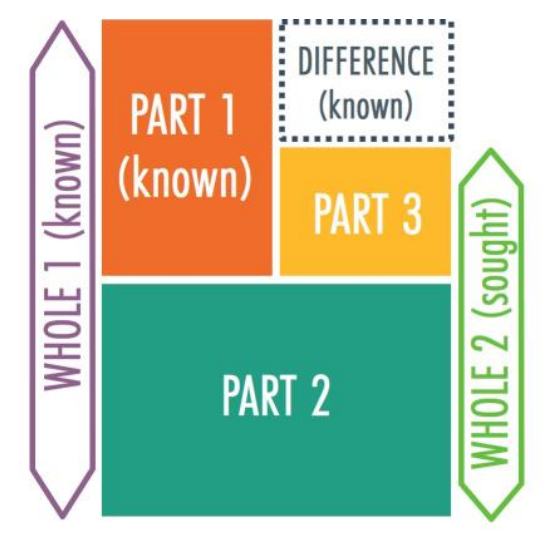

Fig. 1: The mathematical structure of the problems.

The authors argued that the reason behind this difference in participants' ability to use the shortest 1-step algorithm on all three problems was that the quantities used (family members, price, age) evoked different aspects of world knowledge that emphasized either the cardinal or the ordinal nature of the problems' values. The interpretation was that price or family problems tend to be encoded as sets of unordered, disconnected elements that can be grouped and whose values can be added with no ontological order (Gamo et al., 2010). Such an encoding would lead participants to calculate the individual value of each part making the whole, and then add them up, resulting in a 3-step algorithm. On the other hand, in age situations, the age values are inherently ordered and can be represented as states (positions on an axis) and transitions (intervals on a timeline). According to the authors, this type of encoding would make it easier to compare two sets sharing a common part: if Jean and Antoine started attending painting classes at the same age, and Antoine attended the class for 2 years less than Jean, then making the inference than Jean was 2 years younger than Antoine at the end of their respective classes seems especially natural.

\section{Current study}

Going beyond this preliminary work, our ambition for this paper is twofold. First, we aim at showing the critical influence that the distinction between cardinality and ordinality has on human understanding of situations involving numbers. We intend to demonstrate that, more than a question of learning how to enumerate collections, this is an ontological issue regarding the way numerosity is perceived. Second, we intend to build on this semantic 
distinction to investigate adults' representations in the course of mathematical reasoning. Namely, we aim at showing that the nature of the entities staged in word problems interferes with their classification, comparison, encoding, recoding and solving, thus presenting a range of effects falling beyond the scope of the current models of arithmetic problem solving. We designed a series of 6 experiments scrutinizing how the cardinal versus ordinal aspects of numerical situations influence adults' reasoning in a variety of tasks.

\section{Overview of the experiments}

We intend to show that the selection of a specific type of quantity to create a problem statement has an influence on the encoding of the problem into either a cardinal or an ordinal representation, which in turn fosters the use of one of the two existing solving algorithms (see Fig. 2 for a graphical summary of this hypothesis). We designed six experiments in order to examine the importance that the cardinal versus ordinal distinction holds in the representation of numerical situations. They were conducted with adults to underline the strength and pervasiveness of the reported effects.

First, we devised a free sorting experiment to show the fundamental influence of the ordinal versus cardinal distinction on participants' spontaneous categorization of problems, while assessing the validity of our choice of materials. Second, we used an analogy identification experiment to determine whether the hypothesized semantic difference between cardinal and ordinal problems would predict how participants perceive the isomorphism between different problem statements. Third, we used a direct comparison task to evaluate the robustness of the second experiment's findings regarding the perception of the analogy between cardinal and ordinal problems. Fourth, we proposed a solving task with cardinal, ordinal and "hybrid" problems (problems with "ordinalized" cardinal quantities) to show that participants' choice of solving strategies depends on the semantics introduced in the problems. Fifth, we used a solvability-assessment task to evaluate whether participants could solve problems whose unique solution was incompatible with their initial encoding of the problems. Sixth, we used a solution-validity-judgment task to assess whether participants' difficulty to find a problem's unique solution in the fifth experiment could be overcome by the presentation of a candidate solution to evaluate. Altogether, these experiments intended to validate the distinction between cardinal and ordinal situations, to show its influence on the encoding of numerical situations even among adults well past their schooling years, and to evaluate whether its influence could be so robust as to interfere with participants' ability to use relatively basic arithmetic notions to solve 1-step arithmetic problems. 


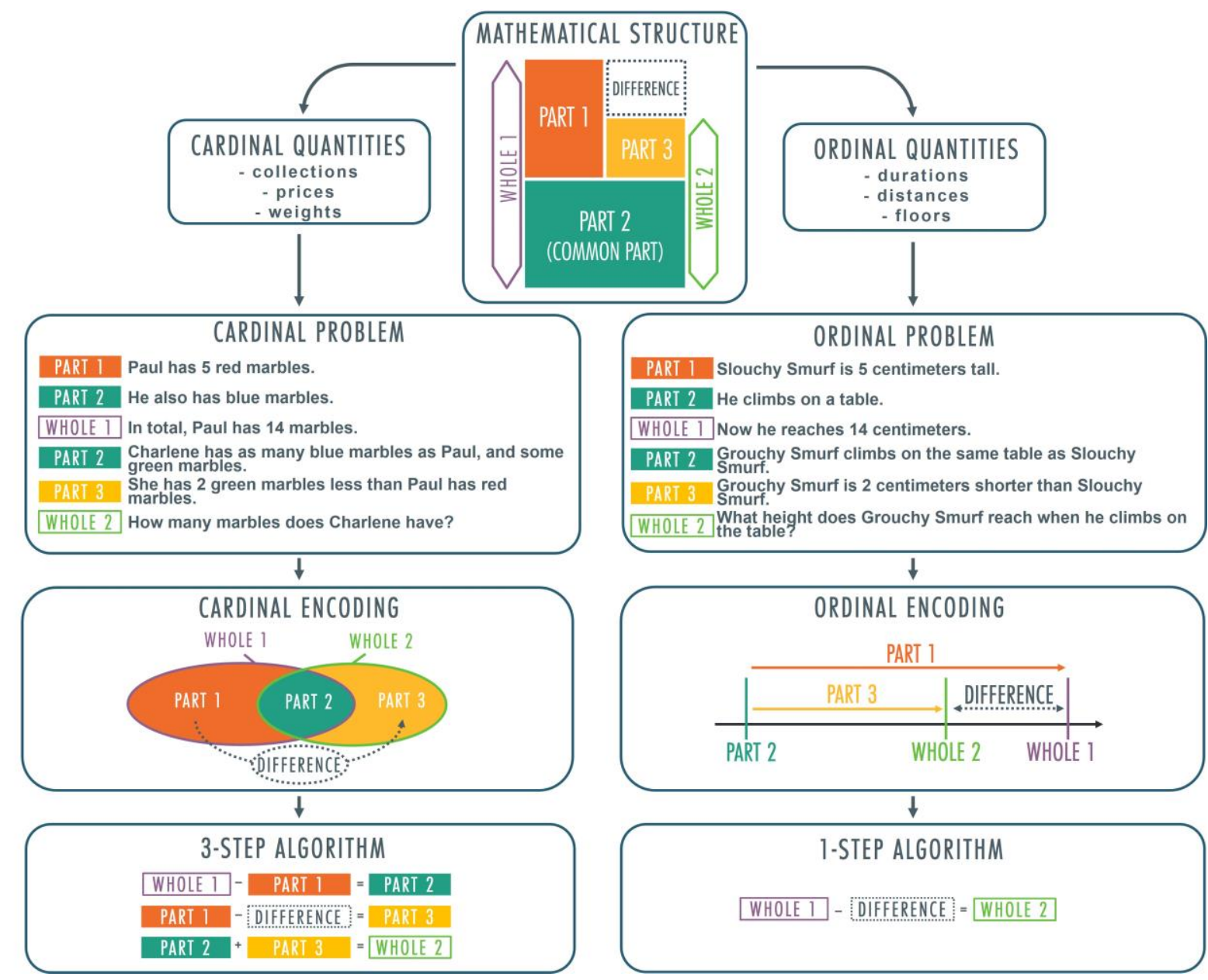

Fig. 2: Graphical summary of the content effects under scrutiny in this paper. Problems sharing the same mathematical structure are implemented with different quantities evoking different encodings, which in turn foster the use of one of the two solving algorithms.

In an attempt to maximize the generality of our findings, we selected 3 types of quantities thought to evoke an ordinal encoding of the situation and 3 types of quantities that we assumed evoked a cardinal encoding. The rationale behind the selection of those specific quantities is presented below; the relevance of this classification will notably be assessed by our first experiment.

\section{Overview of the selected ordinal quantities}

Durations. In English, spatial metaphors expressing durations along a unidimensional space such as "a long time" are prevalent (Casasanto, 2008). The same is true for French, in which the word to designate an extensive period of time corresponds to the literal concatenation of the terms standing for "long" ("long") and "time" (“temps"): “longtemps". In most western cultures, the idea that time evolves along an axis or a timeline is deeply rooted in human understanding of the world (Bonato, Zorzi, \& Umiltà, 2012; Boroditsky, 2011; Droit-Volet \& Coull, 2015; Weger \& Pratt, 2008). Despite cultural differences regarding the direction given to the axis of time (e.g. Fuhrman \& Boroditsky, 2010), the fact remains that time is usually conceived of as a unidirectional axis on 
which values are ontologically ordered.

Heights. Height is a spatial, unidimensional concept. Due to our living in a world where gravity constantly exerts its influence on physical objects, we quickly learn that objects fall in a straight line and we tend to think of height as being an oriented, vertical axis, with a bottom and a top (e.g. Hood, 1998; Hood, Santos, \& Fieselman, 2000; Kim \& Spelke, 1992). Entities' heights can easily be compared or stacked alongside this axis, and there is an ontological order in the values representing the heights of different entities placed atop each other.

Floors. Building floors are stacked on top of each other and they are ordered in a fashion that cannot be altered. If one is to imagine an elevator going from one floor to another, it immediately comes to mind that to go from the $1^{\text {st }}$ floor to the $3^{\text {rd }}$ floor, the elevator must pass the $2^{\text {nd }}$ floor first (Clément $\&$ Richard, 1997).

\section{Overview of the selected cardinal quantities}

Collections. Collections refer to groups of disconnected elements that can be counted as parts of a set. Be it a collection of blue marbles in a bag, a set of iguanas in a terrarium or a group of pupils in a bus, collections of similar elements usually have no ontological order. Fittingly, most studies on the development of cardinality in children's early years resort to tasks consisting in counting collections of objects, such as the Give- $N$ task (e.g. Condry \& Spelke, 2008; Izard, Streri, \& Spelke, 2014; Sarnecka \& Carey, 2008).

Weights. In daily-life, weight is often seen as a property of some definite element, and as such it has no ontological order. For instance, when considering the weight of a stack of dictionaries, it does not matter which one is on top and which one is at the bottom of the stack: computing the total weight simply requires adding the weight of each individual book. Specific weights are assigned to specific entities, and although weight can vary over time, it is rarely encoded along an axis in daily life.

Prices. As with weight, price is usually considered the property of some unordered entities (Gamo et al., 2010). To calculate the total price of a series of items in a store, one usually adds the individual price of each item, in no specific order.

\section{Experiment 1}

The first experiment, a problem sorting task, was designed to assess whether the assumed difference between cardinal and ordinal quantities would lead to different encodings of the problems, reflected by categorization patterns consistent with this distinction. In other words, it intended to provide evidence for the existence of a fundamental distinction between cardinal and ordinal encodings derived from problems sharing an identical deep structure but differing in the quantities used in their problem statements. Indeed, we expected participants' 
categories to reveal the hypothesized difference between cardinal and ordinal problem statements. The experimental design was modeled on the work from Chi, Feltovich, and Glaser (1981), who performed a series of sorting experiments with experts and novices. They showed that, when asked to sort physics problems, novices put together problems sharing similar surface features (e.g. problems featuring pulleys), whereas participants with higher proficiency in physics favored the use of abstract physics principles (e.g. problems that can be solved using Newton's second law) to sort the problems.

Our prediction regarding such problem statements was that the adults' understanding of the problems would neither be strictly limited to surface features, as was the case for Chi et al.'s (1981) lay participants, nor would it be guided by the mathematical deep structure of the problems. Rather, we hypothesized that participants would sort problems depending on how they encode them. We predicted that participants would tend to group together problems evoking a cardinal encoding (collection, price, and weight problems) and that they would group together problems evoking an ordinal encoding (duration, height, and number of floors problems), regardless of the problems' surface features.

\section{Method}

Participants. Participants were recruited on a voluntary basis in the university library. Data collection took place over the course of one week, at the end of which a total of 85 participants had agreed to participate in the experiment (54 women and 31 men, $M=24.31$ years, $S D=8.33$ ). All participants spoke French fluently. None had previously participated in any similar experiment.

Materials and procedure. We created 12 problems: 6 involved cardinal quantities, as previously described ( 2 collection problems, 2 price problems, and 2 weight problems) and 6 featured ordinal quantities ( 2 duration problems, 2 height problem and 2 floors problems). The problems were written in French (original materials for all experiments are available online at https://osf.io/kz6gh/?view_only=2f3fb6b910844e238ae58fef3c61168a. English translation is provided in Tables 2 and 3). The problems all shared the same mathematical structure, they had the same number of sentences and their numerical values were systematically mentioned in the same order across problems. Cardinal and ordinal problems did not significantly differ in number of words; $\mathrm{t}(10)=0.98, p=$ .35 , independent t-test. The goal was to create cardinal and ordinal problems as comparable to each other as possible. The problems were isomorphic, and the numbers used were randomized across problems. The computation of the problems' solving algorithms only required basic mathematical knowledge (additions and subtractions of values below 15) so that the performance differences could not be explained by computational difficulty or lack of knowledge about the arithmetic operations involved. 
Table 2: Cardinal problems used in Experiment 1. The numerical values respected the following rule: $z<4<x$ $<y<15$.

\begin{tabular}{|c|c|c|}
\hline Quantity used & $\mathrm{Pb}$. ID & Problem statement \\
\hline Weight & $\mathrm{Pb} . \mathrm{A}$ & $\begin{array}{l}\text { A bag of pears weighs } x \text { kilograms. } \\
\text { It is weighed with a whole cheese. } \\
\text { In total, the weighing scale indicates } y \text { kilograms. } \\
\text { The same cheese is weighed with a milk pack. } \\
\text { The milk pack weighs } z \text { kilograms less than the bag of pears. } \\
\text { How much does the weighing scale indicate now? }\end{array}$ \\
\hline Weight & $\mathrm{Pb} . \mathrm{B}$ & $\begin{array}{l}\text { Tom takes a Russian dictionary weighing } x \text { kilograms. } \\
\text { He also takes a Spanish dictionary. } \\
\text { In total, he is carrying } y \text { kilograms of books. } \\
\text { Lola takes Tom's Spanish dictionary and a German dictionary. } \\
\text { The German dictionary weighs } z \text { kilograms less than the Russian dictionary. } \\
\text { In total, how many kilograms is Lola carrying? }\end{array}$ \\
\hline Price & $\mathrm{Pb} . \mathrm{C}$ & $\begin{array}{l}\text { In the first meal on the menu, there is a chocolate cake costing } x \text { euros. } \\
\text { The meal also includes an omelet with mushrooms. } \\
\text { In total, the first meal costs } y \text { euros. } \\
\text { In the second meal on the menu, there is the same omelet with mushrooms, and an apple } \\
\text { pie. } \\
\text { The apple pie costs } z \text { euros less than the chocolate cake. } \\
\text { How much does the second meal cost? }\end{array}$ \\
\hline Price & $\mathrm{Pb} . \mathrm{D}$ & $\begin{array}{l}\text { In the stationery shop, Antoine wants to buy a } x \text {-euro ruler. } \\
\text { He also wants a notebook. } \\
\text { In total, that will cost him } y \text { euros. } \\
\text { Julie wants to buy the same notebook as Antoine, and an eraser. } \\
\text { The eraser costs } z \text { euros less than the ruler. } \\
\text { How much will Julie have to pay? }\end{array}$ \\
\hline Collection & $\mathrm{Pb} . \mathrm{E}$ & $\begin{array}{l}\text { Paul has } x \text { red marbles. } \\
\text { He also has blue marbles. } \\
\text { In total, Paul has } y \text { marbles. } \\
\text { Charlene has as many blue marbles as Paul, and some green marbles. } \\
\text { She has } z \text { green marbles less than Paul has red marbles. } \\
\text { How many marbles does Charlene have? }\end{array}$ \\
\hline Collection & $\mathrm{Pb} . \mathrm{F}$ & $\begin{array}{l}\text { Sarah owns } x \text { goldfish. } \\
\text { Her other pets are all iguanas. } \\
\text { In total, she owns } y \text { pets. } \\
\text { Bobby is pet-sitting Sarah's iguanas during the holidays, he puts them with the turtles he } \\
\text { owns. } \\
\text { Bobby owns } z \text { turtles less than Sarah owns goldfish. } \\
\text { How many pets are there at Bobby's? }\end{array}$ \\
\hline
\end{tabular}

Table 3: Ordinal problems used in Experiment 1. The numerical values respected the following rule: $z<4<x$ $<y<15$.

Quantity used $\quad \mathrm{Pb}$. ID
The construction of the cathedral took $x$ years.
Before constructing it, the plans had to be made.
The construction of the cathedral was completed in year $y$.
The construction of the castle started at the same time as the construction of the cathedral.
The construction of the castle took $z$ years less than the construction of the cathedral.
When was the construction of the castle completed?"




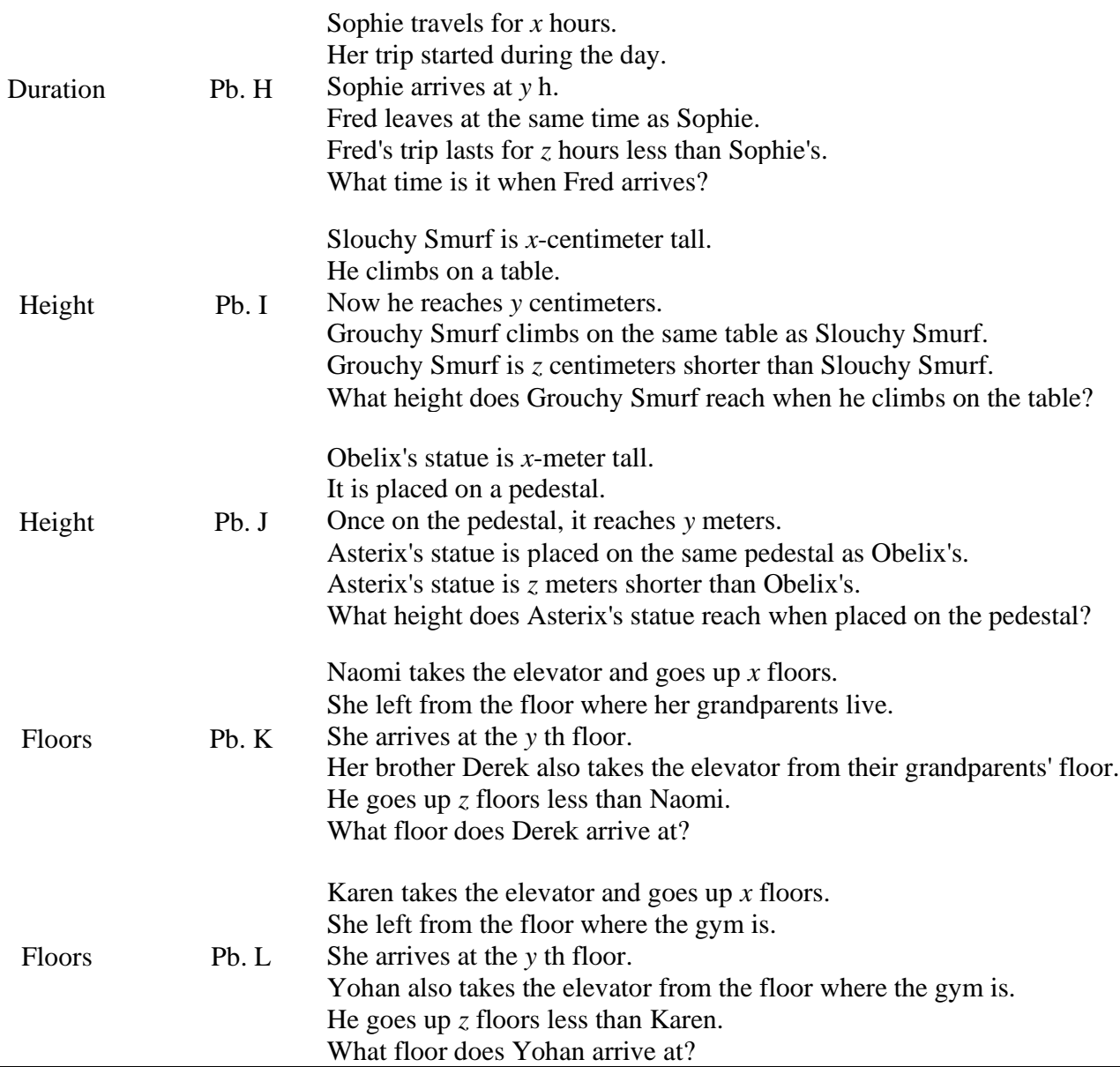

In this experiment, each of the 12 word problems was printed on a separate card. As in Chi et al.'s (1981) experiment, the task was to sort the problems into groups based on similarities of solving strategies. The following instructions were given to the participants:

Here are 12 arithmetic word problems. Please read and study carefully each of them. Your task is to sort the problems into groups based on similarities of solution. You can make as many groups of problems as you deem necessary. This is not a speed test: take your time to read and understand each of these problems. Translated from French.

The participants were not allowed to use a pen and paper so they could not actually solve the problems in order to sort them. Participants all completed the task in less than an hour.

\section{Results}

The categories created by each participant were coded with a co-occurrence matrix describing how many times two problems were sorted together within the same category. A proximity matrix was then elaborated based on the co-occurrence matrix, describing the average perceived proximity between each problem (see Fig. 3). This 
matrix specifies which problems were sorted together most frequently; the higher the value between two problems, the higher the proportion of participants who considered these two problems similar. We tested the hypothesis that problems evoking a similar encoding (either ordinal-ordinal pairs or cardinal-cardinal pairs) would be categorized together more frequently than problems whose encoding is dissimilar (pairs made of one cardinal and one ordinal problem). Results showed that cardinal-cardinal pairs were given a higher proximity score on average $(M=0.90, S D=0.03)$ than cardinal-ordinal pairs $(M=0.75, S D=0.02) ; \mathrm{t}(49)=21.31, \mathrm{p}<.001$, independent $\mathrm{t}-$ test. Similarly, ordinal-ordinal pairs were attributed a higher proximity score on average $(M=0.91, S D=0.04)$ than cardinal-ordinal pairs; $\mathrm{t}(49)=20.60, \mathrm{p}<.001$, independent $\mathrm{t}$-test. In fact, the perceived proximity between any pair of problems whose hypothesized encoding is similar (ordinal-ordinal or cardinal-cardinal) was systematically higher than the perceived similarity between any pair of problems whose hypothesized encoding is dissimilar (cardinal-ordinal).

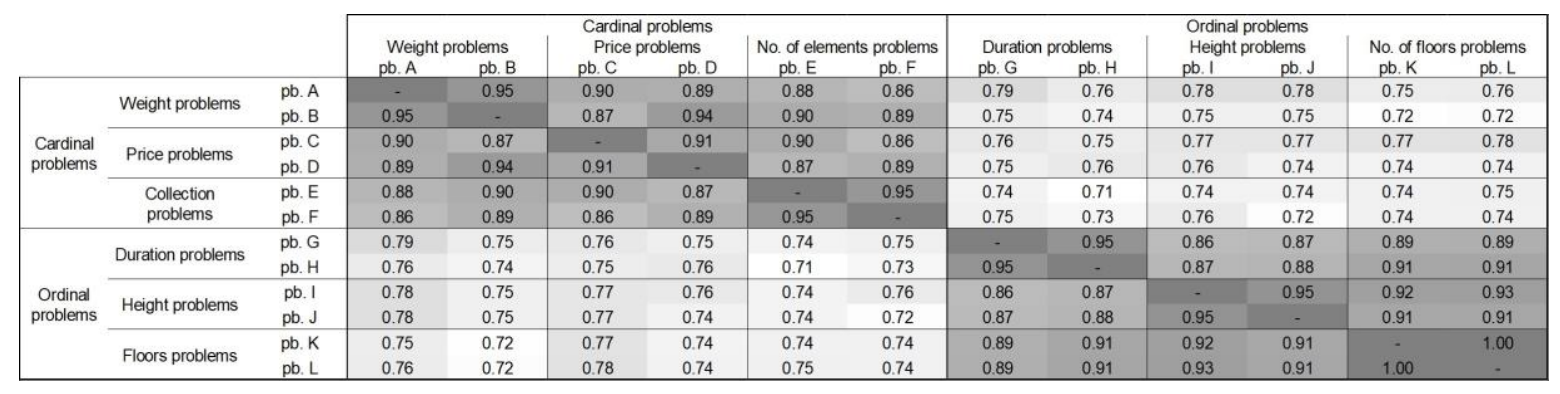

Fig. 3: Co-occurrence proximity matrix between problems. A higher number (and darker hue) indicates a higher co-occurrence frequency.

To better understand participants' classification patterns, we undertook a hierarchical cluster analysis displaying the global taxonomy of the collected categories, using the R package pvclust (Suzuki \& Shimodaira, 2006). Fig. 4 details the clusters appearing in the dataset. For each cluster, the Approximately Unbiased (AU) p-value can be interpreted as follows: if $\mathrm{AU}>.95$, the hypothesis that "the cluster does not exist" can be rejected at the significance level of .05 . This suggests that these clusters do not reflect clustering noise and may be observed in a stable manner. 


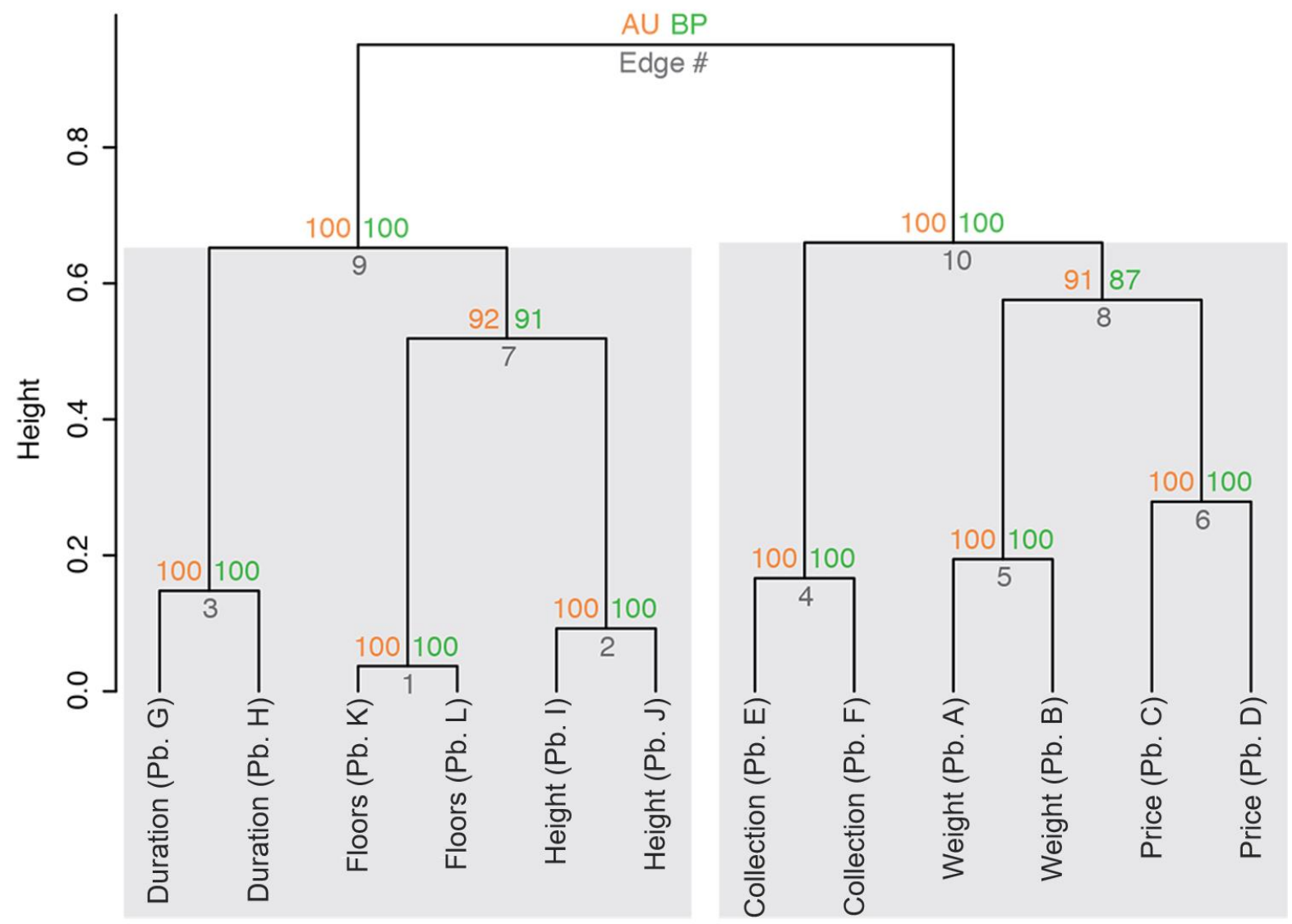

Fig. 4: Cluster dendrogram of the problems. Values are Approximately Unbiased (AU) p-values (Red, left) computed by multiscale bootstrap resampling, Bootstrap Probability $(B P)$ values (green, right) computed by normal bootstrap resampling, and cluster labels (grey, bottom). Clusters with $A U \geq 95$ are highlighted by the grey rectangles and are considered to be strongly supported by the data. Number of bootstrap samples = 10,000 .

All the problems sharing the same quantities (e.g. the two weight problems, the two duration problems, etc.) were grouped in the same lower clusters. This was expected since they both evoked the same encoding (either cardinal or ordinal) and shared a certain degree of surface similarity as well (two problems involving objects being weighed, for example, will both use words such as "weight", "scale" or "weighs", increasing the number of identical surface features). Yet, the two higher clusters displaying a significant AU p-value respectively regrouped all the ordinal problems and all the cardinal problems, regardless of their more specific surface features. This result supports the hypothesis that the cardinal versus ordinal distinction guided participants' sorting patterns. Thus, as hypothesized, some aspects of the problem statements that pertain neither to the most specific surface features nor to the deep structure of the problems influenced the classification. The Bootstrap probability (BP) values, although presumably more biased (Suzuki \& Shimodaira, 2006), confirmed this pattern in the data.

\section{Discussion}

This experiment sought to establish that participants' categories were guided by the semantic distinction we 
introduced between cardinal and ordinal quantities. As predicted, the results showed that participants' categories were not just based on the most superficial similarities between problems. Had it been the case, duration problems would not have been considered as closer from number of floors problems than from collection problems, for instance. Instead, participants perceived the similarity between problems fostering a similar encoding: problems evoking sets of unordered elements were grouped together, and so were problems with elements that could be ordered along an oriented axis. Additionally, participants did not perceive that all problems were isomorphic since that would have resulted in one category containing all the problems. Participants here did not rely on the deep structure of the problems to make their categories. Instead, the results supported our hypothesis that weight, price, and collection problems are encoded differently than height, duration, and floor problems, thus substantiating our selection of those two sets of quantities. Here, the cardinal versus ordinal distinction significantly guided the sorting task, thus corroborating the fundamental role of this distinction.

\section{Experiment 2}

To evaluate how cardinality and ordinality interact with one another when different situations are being compared, and to gather converging evidence regarding the influence that this distinction holds on adults' apprehension of numerical situations, our second experiment focused on participants' interpretation of different problems as analogous. Participants were presented with an unsolved word problem and asked to determine whether a series of target word problems could be solved analogously. We tested the hypothesis that participants can more easily perceive an analogy between two isomorphic problems if they feature quantities evoking a similar encoding (two problems emphasizing the cardinal nature of numbers, or two problems emphasizing the ordinal nature of numbers) than if they do not.

\section{Methods}

Participants. Participants were recruited as part of an undergraduate class at the University of Paris VIII. A total of 191 students ( 116 women and 75 men, $M=27.3$ years, $S D=11.9$ ) agreed to take part in the experiment on a voluntary basis. All participants spoke French fluently. None had previously participated in any similar experiment.

Materials and procedure. In this experiment, we used the same problems as those created for Experiment 1, with the addition of one cardinal problem and one ordinal problem. Each participant was given a 4-page booklet. On the first page, the instructions read:

Below is an arithmetic word problem. Please read it and then study carefully the problems presented on the 
next page. Note, for each of them, if they can be solved using the same solving principle as the problem presented below. This is not a speed test: take your time to read and understand each of these problems.

\section{Translated from French.}

On the same page, a problem statement was printed ("problem A"). This problem was either a cardinal problem (a collection problem) or an ordinal one (a duration problem). On the following page, 6 target problems were presented: 3 ordinal problems (duration, height, floors), and 3 cardinal problems (collection, price, weight). Next to each problem, the participants had to circle their answer "yes" or "no" to the question "can this problem be solved similarly to problem A?". The next two pages had the same setup with different problem statements, where "problem A" was replaced by "problem B", that was either a collection problem (if "problem A" had been a duration problem) or a duration problem (if "problem A" had been a collection problem), and six new target problems. The order of problems A and B was randomized between booklets, as was the order in which the target problems were presented. Participants were not given additional paper to write on, to discourage them from engaging in the resolution of every problem before making their choice. Participants all completed the task in less than an hour.

We predicted that the participants' answers would depend on the similarity between the type of representations fostered by the source and target problems. Namely, participants should perceive the analogy between two cardinal problems or between two ordinal problems more easily than between a cardinal and an ordinal problem. This should translate into an interaction between the cardinal versus ordinal nature of the source problem and that of the target problems.

\section{Results}

We computed the rate of detection of similarity between the source and the target problems, depending on the nature of the quantities involved (see Fig. 5). A two-way repeated measures ANOVA was conducted on the rate of perceived similarity with nature of the quantity in the source problem (ordinal or cardinal) and nature of the quantity in the target problem (ordinal or cardinal) as within factors. As expected, there was no main effect of the cardinal or ordinal semantics attached to the source problem $\left(F(1,190)=1.07, p=.30, \eta_{p}{ }^{2}=.01\right)$ nor of the semantics attached to the target problem $\left(F(1,190)=0.10, p=.08, \eta_{p}{ }^{2}<.01\right)$. There was, however, a significant interaction effect between the two factors, indicating that cardinal target problems were more likely to be selected by the participants when a cardinal source problem was presented, and that ordinal target problems were more likely to be chosen when the source problem was ordinal as well $\left(F(1,190)=72.20, p<.001, \eta_{p}{ }^{2}=.28\right)$. In addition to the interaction, we performed 2-by-2 comparisons of the rate of perceived similarity between cardinal 
and ordinal target problems, depending on the semantics imbued in the source problem. Results showed that cardinal target problems were judged analogous to cardinal source problems significantly more often than ordinal target problems $(82.4 \%$ for cardinal-to-cardinal analogy; $60.9 \%$ for cardinal-to-ordinal analogy; $t(190)=6.50, p$ $<.001, \eta_{p}{ }^{2}=.18$, paired t-test). Similarly, ordinal target problems were judged analogous to ordinal source problems significantly more often than cardinal target problems ( $84.8 \%$ for ordinal-to-ordinal analogy; $62.1 \%$ for ordinal-to-cardinal analogy; $\mathrm{t}(190)=7.25, \mathrm{p}<.001, \eta_{p}{ }^{2}=.22$, paired t-test). In other words, participants identified collection source problems as analogous to other cardinal problems more frequently than they did to ordinal target problems. Reciprocally, the duration source problems were more frequently perceived as analogous to other ordinal problems than to cardinal problems.

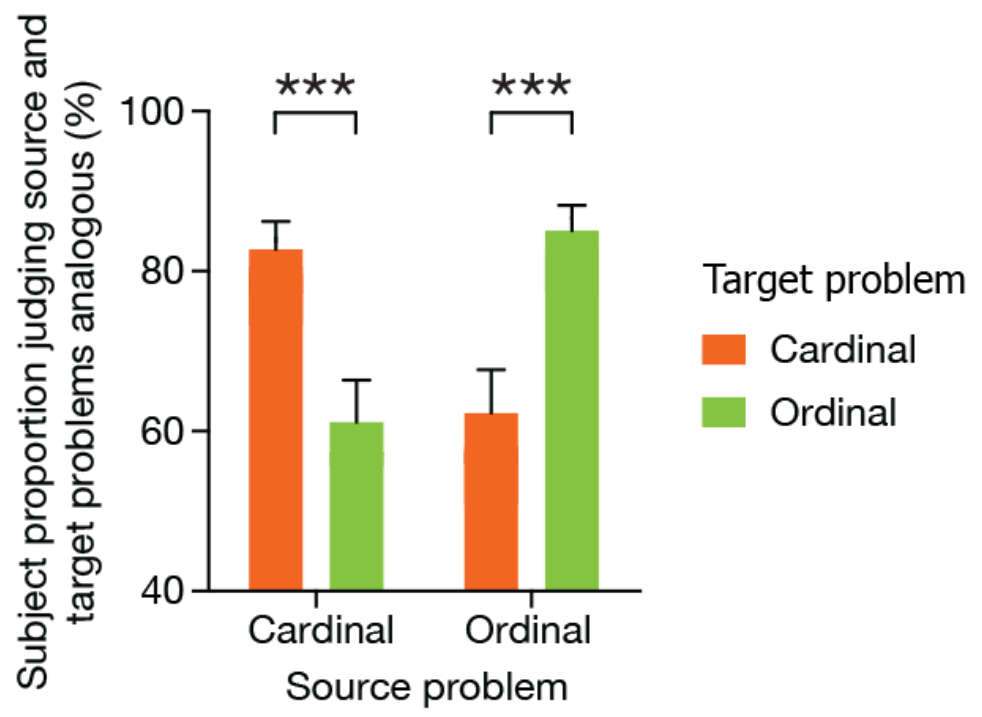

Fig. 5:Rate of perception of the analogy between the source and target problems, depending on the nature of their quantities. Vertical bars denote 95 confidence intervals. $* * * p<.001$, paired $t$-test.

Overall, the analogy rate was higher when the source and target problems both evoked a similar encoding (either two cardinal encodings or two ordinal encodings) than when they evoked dissimilar encodings (a cardinal encoding and an ordinal encoding). Our hypothesis regarding the influence of the encoding on the detection of similarity of solving pattern was thus supported: participants' decisions were significantly influenced by the semantics attached to the quantities used in the problems.

\section{Discussion}

With this experiment, we showed that the encoding difference between cardinal and ordinal problems influences the perception of analogies between isomorphic problems. Participants had significantly more difficulties identifying that two problems shared the same solving principle when these problems featured elements evoking different aspects of their knowledge about the world. This corroborates our claim that general abstract semantic 
properties, such as cardinality or ordinality, play a crucial role in the encoding of mathematical word problems. Depending on the semantics evoked by the problems, participants encode different representations. The representations in turn limit or foster participants' identification of the analogous relations between the problems. Our results support the idea that participants encode a representation whose nature depends on the world knowledge evoked by the problem statement.

While the interaction between the cardinal versus ordinal nature of the target and source problems indicates that participants' encoding of the problems was significantly influenced by the quantities they featured, it might be that participants could have overcome this inability to perceive the similarity between cardinal and ordinal problems if they had tried to directly map the structures of the problems to one another. In other words, suppose participants were encouraged to compare one specific target problem with the source problem instead of simply being asked to select the analogous problems among a series of potential candidates. Would they go beyond their initial encoding of the situation and identify the isomorphism between the problems? We designed a third experiment to answer this question.

\section{Experiment 3}

Experiment 3 aimed at replicating Experiment 2's findings and assessing their robustness by directly presenting pairs of problems to the participants, instead of asking them to identify among a list of target problems the ones that shared a solution principle with the source problem. We hypothesized that the effect observed in Experiment 2 could be replicated in a one-to-one comparison setting, in which participants are encouraged to directly contrast two problem statements. We assumed that when the source and the target problems featured the same type of quantity - cardinal or ordinal - participants would acknowledge the solution equivalence more often than when problems featured different types of quantity.

\section{Methods}

Participants. This experiment was conducted online, on the survey platform Qualtrics. Survey link was sent through social networks and by email. We decided to keep the survey open for one week. After one week, a total of 147 adults participated in the experiment (60 women and 87 men, $M=30.0$ years, $S D=11.5$ ). We estimated that the sample collected was close enough to our target sample size. All participants spoke French fluently. None had previously participated in any similar experiment. The results of five participants were removed since they failed to provide an answer to one or more questions in the experiment. The analyses bear on the 142 remaining participants (59 women and 83 men, $M=29.1$ years, $S D=10.2$ years). 
Materials and procedure. This experiment was conducted on the Qualtrics platform for online experiments. The source and target problems were the same as those used in Experiment 2. On the first page, the instructions read:

Below is an arithmetic word problem. Please read it carefully. On the next pages, you will be presented with a series of arithmetic problems. Indicate, for each new problem, whether it can be solved with the same solving principle as the problem presented below. This is not a speed test: take your time to read and understand each of these problems. Translated from French.

A source problem was then presented, evoking either a cardinal or an ordinal encoding. The following 6 pages repeated the source problem, and then presented a new problem below. Each time, the following question was displayed: "Can these two problems be solved using a similar solution principle?". After 6 target problems had been introduced, a new source problem was presented (a cardinal problem if the first target problem was ordinal, an ordinal problem if the first target problem was cardinal), with the same instructions as before, successively followed by 6 new target problems, one at a time. The source and target problems were the same as those used in Experiment 2. Participants all completed the task in less than an hour.

\section{Results}

For each type of source problem, we analyzed the percentage of participants answering that the source and target problems might be solved following a similar solution principle (see Fig. 6). A two-way repeated measures ANOVA was conducted on participants' rate of identified similarity, with nature of the quantity in the source problem (cardinal or ordinal) and nature of the quantity in the target problem (cardinal or ordinal) as within factors. As in Experiment 2, there was no main effect of the cardinal or ordinal semantics attached to the source problem $\left(F(1,140)=2.14, p=.15, \eta_{p}^{2}=.02\right)$ nor of the semantics attached to the target problem $(F(1,140)=0.41, p=.53$, $\left.\eta_{p}^{2}<.01\right)$. However, we replicated the interaction observed in Experiment 2 between the semantic nature of the source problems and that of the target problems $\left(F(1,140)=73.39, p<.001, \eta_{p}^{2}=.34\right)$. 


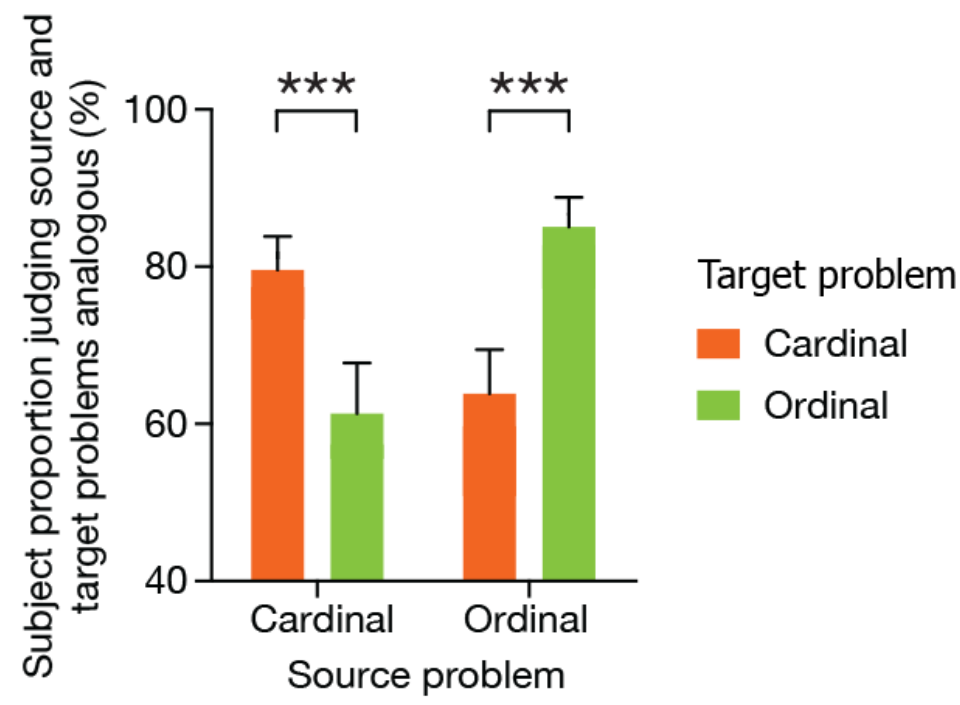

Fig. 6: Rate of perception of the analogy between the source and target problems, depending on the nature of their quantities. Vertical bars denote .95 confidence intervals. $* * * p<.001$, paired t-test.

In addition to the interaction, 2-by-2 analyses revealed that cardinal target problems were judged analogous to cardinal source problems significantly more often than ordinal target problems $(80.9 \%$ for cardinal-to-cardinal analogy; $62.6 \%$ for cardinal-to-ordinal analogy; $\mathrm{t}(140)=5.51, \mathrm{p}<.001, \eta_{p}{ }^{2}=.18$, paired $\mathrm{t}$-test). Similarly, ordinal target problems were judged analogous to ordinal source problems significantly more often than cardinal target problems $(87.7 \%$ for ordinal-to-ordinal analogy; $65.7 \%$ for ordinal-to-cardinal analogy; $\mathrm{t}(140)=6.91, \mathrm{p}<.001$, $\eta_{p}{ }^{2}=.25$, paired t-test). In other words, despite participants being encouraged to directly compare one source problem with one target problem, the problems remained classified as analogous based on the semantics they carried.

\section{Discussion}

In this experiment, it was showed that the effect of the distinction between cardinal and ordinal quantities on the encoding of the situations described in the problems was not altered by the direct presentation of two problems side by side. As predicted, participants had significantly more difficulties identifying that ordinal-cardinal pairs of problems could be solved in the same way, even though the simultaneous presentation of both problems should have facilitated their mapping. Interestingly, the results of Experiment 2 were replicated in this new experimental setting. Our hypothesis regarding the influence of the cardinal versus ordinal distinction on the detection of similarity of solving pattern was thus supported by both experiments. One question that follows from these two experiments regards the role of these robust encoding mechanisms in the choice of a solving algorithm. To what extent do the constructed representations dictate participants' solving strategies? By using problems designed to evoke cardinal, ordinal, or hybrid encodings, the next experiment was designed to evaluate how these encoding 
differences may influence participants' choice of an algorithm in a more traditional solving task.

\section{Experiment 4}

The goal of this fourth experiment was twofold. First, we intended to demonstrate that participants' ability to use a specific solving strategy directly depended on the nature of their semantic encoding of the problem. Second, we aimed at showing that by changing the semantics imbued in a problem statement, we could significantly alter its encoding on the cardinal versus ordinal dimension. To this end, we introduced a new type of problems, in addition to the cardinal and ordinal problems used in the previous experiments. We called those new problems "hybrid", as they were meant to elicit an ordinal encoding using cardinal quantities. Indeed, those hybrid problems involved only cardinal quantities (price, weight, collection) but their problem statements featured a scenario fostering an ordinal encoding by describing how the cardinal quantities changed over time. For instance, hybrid weight problems were created by describing the weight of a baby growing over time in order to favor an ordinal representation of the weight. We predicted that introducing those characteristics without changing the quantities themselves would influence the encoding of the problems and the algorithms subsequently implemented.

Since we instructed participants to solve the problems using as few operations as possible, our main hypothesis regarded the rate of use of the 1-step algorithm. We predicted that problems involving ordinal quantities would lead to a greater use of the 1-step algorithm than problems involving cardinal quantities, due to the ordinal encoding making it easier to perceive the validity of this solution. Second, we hypothesized that hybrid problems would lead to a significantly higher rate of 1-step algorithm than cardinal problems, due to the ordinal semantics attached to the problem statements. Additionally, we aimed at assessing whether hybrid problems would be solved by the 1-step algorithm as often as ordinal problems, or not.

\section{Method}

Participants. Participants were recruited on a voluntary basis among the undergraduate population of three universities. A total of 181 students participated in this experiment after giving informed consent (123 women and 58 men, $M=23.35$ years, $S D=7.82$ ). All participants spoke French fluently. None had previously participated in any similar experiment.

Materials and procedure. A pool of 18 word problems was used for this experiment: the same 12 problems ( 6 cardinal and 6 ordinal) as in the first three experiments, and 6 new hybrid problems (see Table 4 for a selection of hybrid problems). Each participant saw 9 problems in total: 3 of each category.

Table 4: Example of hybrid problems. The numerical values respected the following rule: $z<4<x<y<15$. 


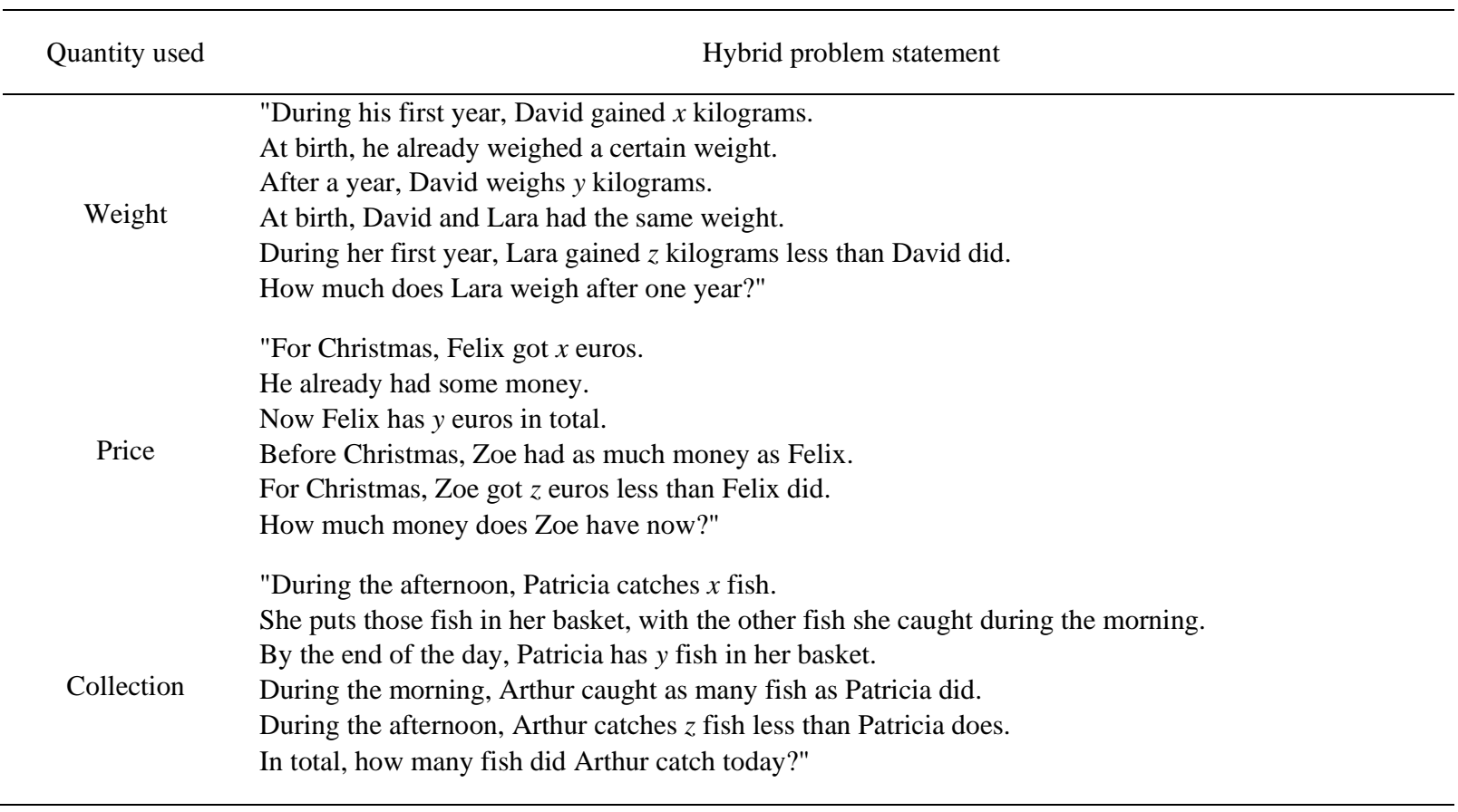

The participants all received 10-page booklets with instructions printed on the first page. The instructions read:

You will find an arithmetic problem on each page of this booklet. Your task is to solve the problems using as few operations as possible. You can use the 'draft' area, but please copy in the 'response' area all the operations that you used to come up with the solution. This is not a speed test: take your time to read and understand each of these problems. Remember that the goal is to solve the problems using as few operations as possible. For every problem, we ask you to write down every operation(s) that you used to come up with the solution, even the simplest one that you can calculate mentally. For instance, the computation " $15-6-2=7$ ", should not be written as a unique operation, but broken down as " 15 $6=9$ " and "9 $9-2=7$ ", which then counts for two operations. Translated from French.

Problem order was randomized across booklets. Each page in the booklet was divided in three parts: the problem statement, the "draft" area and the "response" area. Participants all completed the task in less than an hour.

Scoring. A problem was considered as correctly solved when the obtained result came with the appropriate calculations. The strategies leading to success were categorized either as correct 1-step algorithm or as correct 3step algorithm. When the written operations were correct and the written solution was within $+/-1$ of the correct result, this was deemed a calculation error and problems were still considered as correctly solved. Other answers were considered as false.

\section{Results}

The percentage of correct solving using both algorithms was calculated. Fig. 6 details the rate of use of the 1-step 
algorithm for each problem category. A one-way repeated measures ANOVA was conducted on the rate of use of the 1-step algorithm between cardinal, hybrid and ordinal problems. Results indicated that there was a main effect of problem category (cardinal/hybrid/ordinal) $\left(F(2,360)=52.13, p<.001, \eta_{p}{ }^{2}=.22\right)$. Three paired sample t-tests were used to compare the different conditions, with Bonferroni adjustment for multiple comparisons. As predicted, cardinal problems led to a significantly lower rate of 1-step algorithm $(M=0.28, S D=0.39)$ than ordinal problems $(M=0.47, S D=0.39) ;(t(180)=9.34, p<.001, d=0.48)$, which supported the hypothesis that the use of cardinal versus ordinal quantities significantly influenced the encoding of the problem statements, and subsequently shaped the solving algorithms used by the participants. Moreover, as hypothesized, the 1-step algorithm was more frequently used on hybrid problems $(M=0.38 S D=0.40)$ than on cardinal problems; $t(180)$ $=5.91, p<.001, d=0.24$. This result showed that, by giving specific semantic properties to a problem statement, it was possible to influence the encoding and manipulate which solving algorithm participants would use. A semantically cardinal quantity presented in an ordinal context could thus lead more often to the encoding of an ordinal representation than a cardinal quantity presented in a context that does not present ordinal features.
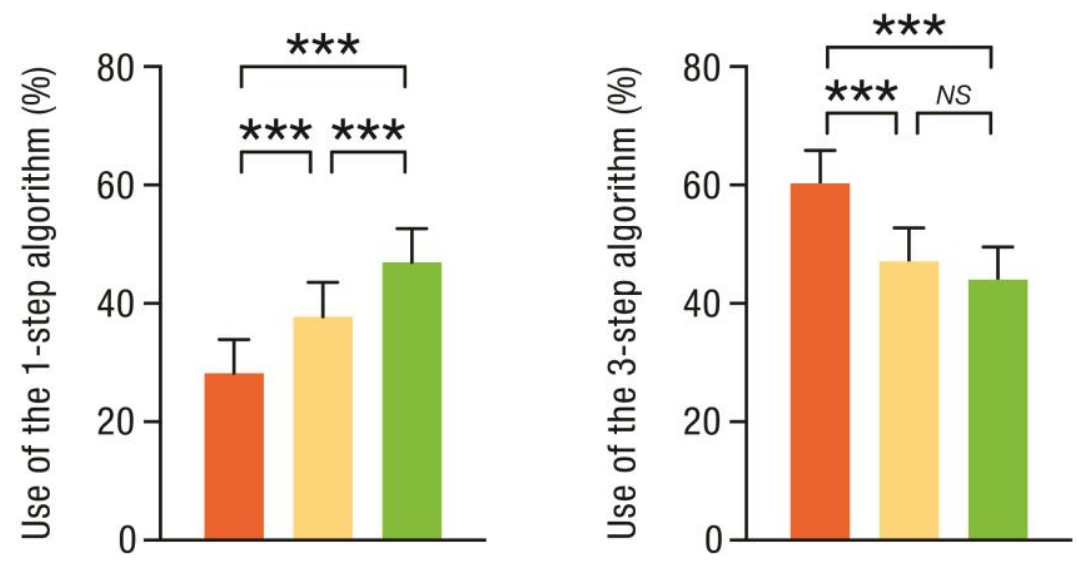

Semantics attached
to the problems
Cardinal
Hybrid
Ordinal

Fig. 6: Rate of use of the 1-step algorithm and 3-step algorithm, depending on the semantics attached to the problems. Vertical bars denote .95 confidence intervals. $* * * p<.001$ (paired t-test).

Interestingly, the comparison between hybrid problems and problems with ordinal quantities revealed that the rate of use of the 1-step algorithm was still higher on ordinal problems $(M=0.47, S D=0.39) ; t(180)=4.93, p<.001$, $d=0.23$. This seems to indicate that, while the manipulation that was performed to "ordinalize" cardinal quantities had a significant impact when compared to standard cardinal problems, an "ordinalized" quantity remained less ordinal than a typical ordinal one.

Regarding the rate of use of the 3-step algorithm, we performed a one-way repeated measures ANOVA to evaluate if it differed between cardinal, hybrid and ordinal problems. Results indicated the presence of a main effect of problem category (cardinal/hybrid/ordinal) on the use of this algorithm as well $(F(2,360)=27.80, p<$ 
$.001, \eta_{p}{ }^{2}=.13$ ). We used paired sample t-tests to perform pairwise comparisons between the three conditions, with Bonferroni adjustment for multiple comparisons. Results showed that participants resorted to the 3-step algorithm more often on cardinal problems $(M=0.60 S D=0.39)$ than on ordinal problems $(M=0.44 S D=0.38)$; $\mathrm{t}(180)=6.79, \mathrm{p}<.001$. Interestingly, they also used the 3-step algorithm more often on cardinal than on hybrid problems $(M=0.47 S D=0.38) ; \mathrm{t}(180)=5.80, \mathrm{p}<.001$. This is in line with the idea that introducing cardinal quantities in an ordinal context could help "ordinalize" participants' representation. There was, however, no significant difference between the rate of use of the 3-step algorithm on hybrid and on ordinal problems; $t(180)=$ $1.38, \mathrm{p}=.17$

\section{Discussion}

In this experiment, the analysis of the solving algorithms provided cues on how the encoding of the problems influences participants' solving strategies. Participants' use of the shortest algorithm was dependent on the type of quantities involved in the problem statement. Despite participants being explicitly instructed to use as few operations as possible to solve the problems, they mostly used the 3-step algorithm to solve the cardinal problems. This experiment further supported the claim that the ordinal versus cardinal dimension was the main factor constraining algorithm choice. Indeed, a change in strategy choice followed the "ordinalization" of cardinal quantities, thus showing that the ability to use the 1-step algorithm was directly dependent on how much the problem statement emphasized the ordinal nature of its numerical values.

So far, we have studied how arithmetic word problems are initially encoded and how the interpreted representations in turn influence the solving algorithms used. However, as mentioned in the introduction, the initial encoding of a problem does not always provide a solution to the solver. We then investigated whether participants can overcome their initial representation of the problems when the one they first encoded does not lead to a solution. That is, we created the conditions to explore the difference between situations in which one has to construct a new representation of the situation presented, and those in which there is no need for a new representation to be constructed. Such a recoding process would imply to disregard the cardinal semantics evoked by specific quantities, and to construct a new encoding of the situation regardless of their influence.

\section{Experiment 5}

In this fifth experiment, we designed situations meant to have participants construct a representation leading to a dead end. In other words, we created problems for which the initial encoding would not provide a successful solving algorithm, thus hindering the solving process. The problems could nonetheless be solved if participants 
constructed a different encoding of the situation. We tested the participants' proficiency to use the 1-step solving algorithm on problems that would spontaneously elicit the 3-step algorithm. For that purpose, cardinal problems that only featured two numerical values were introduced, making the 3-step solving algorithm impossible to use. By contrast, the 1-step algorithm was still efficient for reaching the solution with the two remaining numerical values.

A solvability judgment task requested participants to tell whether problems were solvable and to write down the solution of the solvable problems. Our aim was to show that semantic constraints associated with cardinal situations would lead adult participants to evoke an encoding incompatible with the 1-step algorithm, leading them to fail to find the solution to the problems. We expected them to incorrectly dismiss a perfectly valid solving algorithm, erroneously labeling a 1-step subtraction problem as unsolvable. We also expected that succeeding in solving cardinal problems would require an extra representational step, since the initial representation favors the use of the 3-step algorithm. We assumed that the construction of a new representation would be costly and time consuming. Therefore, our predictions were twofold: first, we hypothesized that participants would incorrectly reject the cardinal solvable problems more often than the ordinal solvable problems because of the conflict between a cardinal representation and the 1-step algorithm. Second, when correctly solved, cardinal problems would require a significantly longer response time than correctly solved ordinal problems, because of the extra step needed to build a new representation compatible with the 1-step algorithm.

\section{Method}

Participants. This experiment was conducted online, on the survey platform Qualtrics. Survey link was sent through social networks only. The survey was kept open for one week, after which a total of 89 adults had participated on a voluntary basis ( 50 women and 39 men, $M=32.1$ years, $S D=13.4$ years). All participants spoke French fluently and none had previously participated in any similar experiment. Because part of the analyses were performed on response times, we removed 15 participants who either mentioned taking a break during the test or who answered at least one of the questions in less than 5 seconds (which meant they either mis-clicked or did not take the time to read the problem). The analyses bear on the 74 remaining participants (44 women and $30 \mathrm{men}, M$ $=33.8$ years, $S D=13.4$ years).

Materials and procedure. The problems used in this experiment were similar to the ones in previous experiments, except for the value of Part 1 (see Fig. 1) that was removed from the statements so that the 3-step algorithm could not be used anymore. Consequently, the only way to solve the problems was to resort to the 1step algorithm, which required using the remaining values of Whole 1 and of the Difference (see Fig. 1). Table 5 
presents 6 examples of such problems ( 3 cardinal and 3 ordinal problem statements) created from the ones used in the previous experiments by removing the $x$ value corresponding to Part 1 . Ordinal problems were 333.5 characters long on average $(S D=38.37)$ and cardinal problems were 304 characters long on average $(S D=$ 44.94). This length difference was not statistically significant $(\mathrm{t}(10)=1.18, p=.26$, paired $\mathrm{t}$-test).

Table 5: Example of target problems used in the study. Changes introduced from the problems in experiments 1 to 3 are italicized in the table for the sake of clarity, but they were not made apparent in the experiment. Translated from French.

\begin{tabular}{|c|c|}
\hline Cardinal target problems & Ordinal target problems \\
\hline $\begin{array}{l}\text { Paul has a certain amount of red marbles. } \\
\text { He also has blue marbles. } \\
\text { In total, Paul has } 14 \text { marbles. } \\
\text { Jolene has as many blue marbles as Paul, and some green } \\
\text { marbles. } \\
\text { She has } 2 \text { green marbles less than Paul has red marbles. } \\
\text { How many marbles does Jolene have? }\end{array}$ & $\begin{array}{l}\text { Sofia travelled for a certain time. } \\
\text { Her trip started during the day. } \\
\text { Sofia arrived at } 14 \mathrm{~h} \text {. } \\
\text { Fred left at the same time as Sofia. } \\
\text { Fred's trip lasted } 2 \text { hours less than Sofia's. } \\
\text { What time was it when Fred arrived? }\end{array}$ \\
\hline $\begin{array}{l}\text { In the store, Antoine wants to buy a ruler costing a } \\
\text { certain price. } \\
\text { He also wants a notebook. } \\
\text { In total, that will cost him } 14 \text { dollars. } \\
\text { Julie wants to buy the same notebook as Antoine, and an } \\
\text { eraser. } \\
\text { The eraser costs } 2 \text { dollars less than the ruler. } \\
\text { How much will Julie have to pay? }\end{array}$ & $\begin{array}{l}\text { Slouchy Smurf is a certain height. } \\
\text { He climbs on a table. } \\
\text { He now attains the height of } 14 \text { centimeters. } \\
\text { Grouchy Smurf climbs on the same table as Slouchy Smurf. } \\
\text { Grouchy Smurf is } 2 \text { centimeters shorter than Slouchy Smurf. } \\
\text { What height does Grouchy Smurf attain when he climbs on the } \\
\text { table? }\end{array}$ \\
\hline $\begin{array}{l}\text { Tom takes a Russian dictionary weighing a certain } \\
\text { weight. } \\
\text { He also takes a Spanish dictionary. } \\
\text { In total, he is carrying } 14 \text { kilograms of books. } \\
\text { Lola takes Tom's Spanish dictionary and a German } \\
\text { dictionary. } \\
\text { The German dictionary weighs } 2 \text { kilograms less than the } \\
\text { Russian dictionary. } \\
\text { In total, how many kilograms is Lola carrying? }\end{array}$ & $\begin{array}{l}\text { Katherine took the elevator and went up a certain number of } \\
\text { floors. } \\
\text { She left from the floor where the gym is. } \\
\text { She arrived to the 14th floor. } \\
\text { Yohan also took the elevator from the floor where the gym is. } \\
\text { He went up } 2 \text { floors less than Katherine. } \\
\text { What floor did Yohan arrive to? }\end{array}$ \\
\hline
\end{tabular}

Although our predictions only regarded solvable problems, we also included unsolvable fillers in the materials, so that not every problem had a solution. Among those fillers the value of Part 1 was preserved, and the value of Whole 1 was removed, which made the problems unsolvable with either algorithm. Thus, an equal number of fillers was introduced to achieve a uniform distribution of solvable/unsolvable answers. Problem order and numerical values were randomized between participants. On the first page of the online experiment, the following instructions were written:

You will find an arithmetic problem on each page of this survey. Your task is to identify which problems can be solved and to indicate for each of them the operation you used to solve it, as well as the solution you found. Be careful: some of the problems cannot be solved with the available information, thus your answer in such cases should be 'it is not possible to find the solution'. This is not a speed test: take your time to read and understand each of these problems. Translated from French. 
On each page of the survey, a problem was displayed with the following question below it "Given the data provided, is it possible to find the solution?" and two buttons "Yes" and "No". When the participants pressed "Yes", two new questions appeared, asking them to indicate respectively the operation needed to solve the problem and the result of the operation. Participants used the keyboard to write down their answers. After participants answered all 12 problems, a new page was displayed asking them for their gender, date of birth, and whether they made any breaks during the completion of the experiment. Participants all completed the task in less than an hour.

As previously stated, our first prediction was that participants would perform better on solvable problems with ordinal quantities compared to solvable problems with cardinal quantities. Indeed, we believed that problems whose spontaneous representation was associated with a 3-step algorithm would often lead participants to ignore the 1-step algorithm, due to cardinal representations being incompatible with the shortest algorithm. Our second prediction regarded the cardinal problems that were correctly solved by the participants despite the conflict between a cardinal encoding and the 1-step algorithm. We hypothesized that higher response times would be recorded on successfully solved cardinal problems compared to successfully solved ordinal problems, due to participants needing additional time to overcome their initial encoding of the situations and build a new representation, compatible with the 1-step algorithm.

\section{Results}

The dependent variable was the percentage of correct answers on solvable problems. Ordinal solvable problems were successfully solved in $91.9 \%$ of the trials, and cardinal solvable problems in $68.5 \%$ of the trials (see left graph of Fig. 7). A paired t-test was performed on participants' mean rate of success for cardinal and ordinal problems and showed that the difference was statistically significant $(t(73)=6.38, p<.001, d=0.97)$, therefore supporting our first hypothesis. 

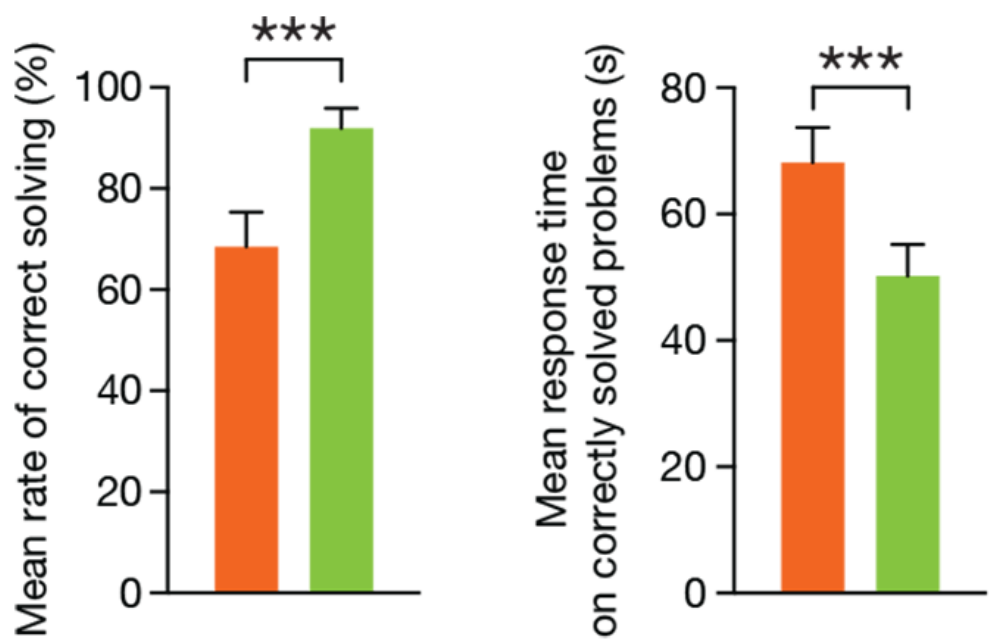

\section{Semantics attached to the problem \\ Cardinal \\ Ordinal}

Fig. 7: Mean rate of correct resolution (left) and mean response time on correctly solved problems (right) depending on the semantic nature of the quantities used in the problems. Vertical bars denote 95 confidence intervals. $* * * p<.001$ (paired t-test).

Response times on correctly solved cardinal and ordinal problems were then compared in order to test our prediction that accessing the correct 1-step algorithm on problems inducing a cardinal encoding incompatible with this algorithm would require higher response times than it would on problems evoking an ordinal representation. On average, participants took 68.7 seconds to successfully solve cardinal problems, and 49.8 seconds for ordinal problems (see right graph of Fig. 7). Because we only considered the response times for correctly solved problems, the number of measures per participant could vary from 0 to 6 , so we resorted to a mixed model analysis instead of a repeated measures ANOVA. We removed 4 participants who did not manage to correctly solve at least one cardinal and one ordinal problems, since no comparison could be made between their response times in both conditions. A linear mixed model with participants as a random factor and problem type (cardinal versus ordinal) as a fixed effect showed that the difference between cardinal and ordinal problems had a significant effect on response times of successfully solved problems $(F(1,69)=20.38, p<.001)$, thus supporting our second hypothesis.

\section{Discussion}

As predicted, it was more difficult for participants to use the 1-step algorithm for cardinal than for ordinal problems, despite the 1-step algorithm being the only remaining possibility to find the solution. Besides, as hypothesized, when participants overcame the difficulty and found the solution to a cardinal problem, it required extra processing time, presumably attributable to the construction of a new representation of the situation.

While the fourth experiment showed that the 1-step algorithm is more frequently used on ordinal than on cardinal problems, this fifth experiment showed that this effect is not the result of a mere preference but, instead, seems to be the consequence of strong limitations imposed by the type of quantity used. In fact, this effect was so 
pervasive that in many cases adult participants failed to see that these one-step subtraction problems could be solved at all. Yet, not all the participants failed, and some of them even managed to use the 1-step algorithm in certain cases. However, in order to overcome the constraints imposed by their world knowledge about the problem's quantities and use a conflicting solving algorithm, the participants had to discard their initial representation and construct a new encoding closer to the problem's mathematical structure. The existence of such a recoding step, akin to a re-representation process (Vicente, Orrantia, \& Verschaffel, 2007), was supported by the longer response times required on correctly solved cardinal problems.

Once participants realize that the 3-step algorithm cannot be used given the available information, they might be tempted to discard these problems as unsolvable and move on. In the $6^{\text {th }}$ experiment, we went a step further and provided participants with a potential solution to the problems, one that they would not usually consider. By giving them such a clue, we were able to assess their difficulty to construct an alternate encoding of the situation even when directly incited to do so.

\section{Experiment 6}

In this experiment, we provided participants with the 1-step solution algorithm of each problem and asked them to directly evaluate its validity. Because of the high failure rates on cardinal problems in Experiment 5, we tested the stronger hypothesis that providing the solution algorithm would not be enough to systematically foster an appropriate encoding of the situation by the participants. We hypothesized that even when explicitly presented with the solution, participants would reject it more often in the cardinal condition than in the ordinal one since their encoding of cardinal problems would conflict with their solution (i.e. promoting an unusable 3-step algorithm instead of the 1-step algorithm). Because of the need to overcome the initial representation, we hypothesized that the correct identification of the solution would require more time for cardinal than for ordinal problems. This experimental paradigm resembled the one used in a recent study we conducted among expert mathematicians (Gros, Sander, \& Thibaut, 2019). However, in the present experiment, participants could take as long as they wished to complete the task, whereas in Gros et al. (2019), participants were explicitly told to solve the problems as fast as possible, with the intended purpose of increasing their error rates. The absence of time constraint was meant to give participants the opportunity to read the problems until they were certain of their decision and to engage in a recoding of their initial representation if need be.

\section{Method}

Participants. This experiment was conducted online, on the survey platform Qualtrics. Survey link was sent 
through a Parisian mailing list for cognitive science experiments. We kept the survey open for one week, after which a total of 223 adults had participated on a voluntary basis. The increase in sample size is attributed to a response rate above our expectations on the mailing list for voluntary participants. All spoke French fluently and none had previously participated in any similar experiment. Among them, 27 were removed from the analysis because they either took a break during the test or answered at least one of the questions in less than 5 seconds (which suggested they did not take the time to read the problem). The analyses were performed on the remaining 196 participants (135 women and 88 men, $M=34.5$ years, $S D=14.8$ years).

Materials and procedure. The only difference between the present experiment and the previous one was the fact that a solution was proposed. Instead of having the participants solve the problems themselves, a solution was proposed for each problem, and they were asked to judge whether the provided solution was valid or whether the problem was unsolvable. For every problem, the question "Given the data provided, is it possible to find the solution?" was displayed. Two choices appeared below: (a) "No, we do not have enough information to solve this problem." and (b) "Yes: numerical value 1 - numerical value $2=$ result. Sentence presenting the result". For instance, on one of the elevator problems, the option (b) was: "Yes: $11-2=9$. Karin arrives at the $9^{\text {th }}$ floor." Participants all completed the task in less than an hour.

\section{Results}

As in Experiment 5, we first analyzed the ratio of correct answers on solvable problems depending on the type of quantities used. The left graph of Fig. 8 shows that, among the target problems, the cardinal ones had a lower success rate $(63.6 \%)$ than the ordinal ones $(88.4 \%)$. A paired t-test performed on the participants' mean rate of success confirmed that this difference was significant $(t(195)=9.25, p<.001, d=0.87)$.
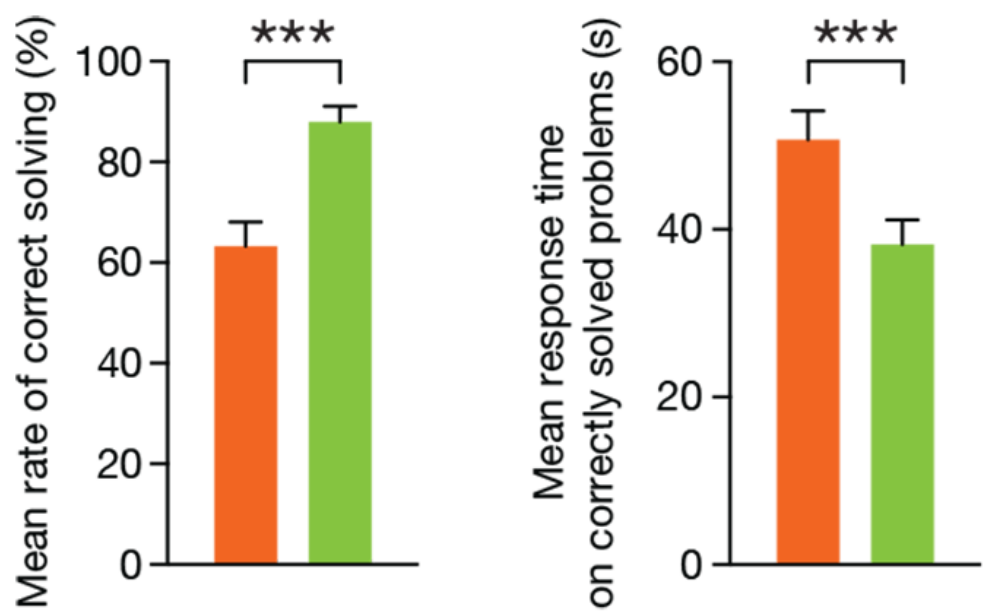

\section{Semantics attached to the problem}

Cardinal

- Ordinal

Fig. 8: Mean rate of correct resolution (left) and mean response time on correctly solved problems (right) 
depending on the semantic nature of the quantities used in the problems. Vertical bars denote 95 confidence intervals. *** $p<.001$.

In order to assess the validity of our second hypothesis, we analyzed the response times of correct answers on the target solvable problems. The 26 participants who did not manage to correctly respond to at least one cardinal and one ordinal problems were removed from this analysis, since no comparison could be made between their response times in both conditions. The right graph of Fig. 8 shows that providing a correct answer required a shorter response time for ordinal (38.6 seconds) than for cardinal problems (51.4 seconds). A linear mixed-model with participants as a random effect and the cardinal versus ordinal distinction as a fixed factor confirmed that the effect was statistically significant $(F(1,169)=30.28, p<.001)$, supporting the second hypothesis.

\section{Discussion}

This experiment, involving a solution validity assessment task, supported the effects observed in the previous one, involving a solution discovery task. The analyses indicated that even when the correct solution was provided, it was more difficult for them to accept it when it was not compatible with the initial encoding of the problem. Despite the problems being solvable with a mere subtraction, participants went so far as to reject the correct solution and dismiss the problems as "unsolvable". Furthermore, overcoming this difficulty required more time, thus supporting our prediction of the need for an extra processing step when faced with an inapt representation. These results suggest that the encoding effects identified in the $5^{\text {th }}$ experiment are not restricted to the elaboration of a solving strategy, but also to the evaluation of its validity. This experiment provides additional evidence that the cardinal versus ordinal distinction constrains the encoding of problems, since even when no solving algorithm had to be produced by the participants, their interpretation precluded them from considering the given solution albeit a single subtraction - as an acceptable one.

\section{General discussion}

\section{The pervasive influence of the cardinal-ordinal distinction}

Taken together, the present six experiments shed light upon the foundational part played by the cardinal versus ordinal distinction in adults' reasoning about numerical situations. The first experiment confirmed the relevance of the selected quantities, as the distinction between cardinal and ordinal quantities drove adults' sorting patterns. Experiments 2 and 3 demonstrated that the encoding difference between cardinal and ordinal problems impacts participants' success in perceiving problems as analogous, even when explicitly instructed to directly compare two problems. The fourth experiment's findings were twofold. First, it showed that the distinction between 
cardinal and ordinal problems influences adults' choice of a solving algorithm. Second, it demonstrated that it is possible to manipulate a problem's semantics by presenting cardinal quantities in a context emphasizing the ordinality of the problem's values. The changes that were introduced to make the cardinal problems more ordinal had a significant effect on participants' algorithm choice, thus bolstering the importance of the cardinal versus ordinal distinction and strengthening the view that the encoding difference observed depended on the semantic dimensions that were manipulated in the problems. The fifth experiment showed that even when only one solution was available, adult participants have difficulties to find it when it is not compatible with what is assumed to be their spontaneous encoding of the problems. They were more likely to erroneously judge that cardinal problems cannot be solved and, when they did find the solution to a cardinal problem, it nonetheless required a longer reasoning time. Finally, the sixth experiment demonstrated that presenting the participants with a potential solution does not suppress the effects observed in Experiment 5, as participants continued to struggle to identify the solution of cardinal problems.

The fact that the distinction between cardinal and ordinal quantifications could exert a pervasive effect on adults' grasp of numerical situations illustrates the foundational nature of this distinction for the human mind. Despite a growing body of research on the comparative development of the notions of cardinality and ordinality, little is known regarding adults' ability to alternatively perceive the cardinality or the ordinality of the numerical situations they encounter, depending on the daily-life knowledge imbued in these situations. Our understanding of mathematics is deeply rooted into our understanding of the world (Fischbein, 1987; Hofstadter \& Sander, 2013; Lakoff \& Núñez, 2000) and, as a result, we tend to apply real-life constraints to abstract mathematical notions. Here, we showed that our perception of ordinality and cardinality in scenes of our daily lives has a profound effect on our ability to engage in mathematical reasoning in these situations.

\section{Implications for mathematical word problem solving}

Although converging works have shown that slight modifications in the wording of otherwise structurally identical mathematical word problems could result in significant performance disparities (Carpenter \& Moser, 1982; Cummins, Kintsch, Reusser, \& Weimer, 1988; De Corte, Verschaffel, \& De Win, 1985; Hudson, 1983; Nesher, Greeno, \& Riley, 1982; Squire \& Bryant, 2002; Thevenot \& Oakhill, 2005), the identification of the underlying reasoning processes accounting for such variations has remained a debated issue to this day. One possible explanation can be found in the schema theory (Kintsch, 1988; Kintsch \& Greeno, 1985; Rumelhart, 1980; Schank, 1975; Schank \& Abelson, 1977), which proposes that our ability to identify the algorithmic solution of a word problem depends on the activation of the appropriate schema in long-term memory. However, a number 
of shortcomings have been shown to hinder the schema theory's explanatory power of the interpretative effects that seem to be central in mathematical word problem solving (Devidal, Fayol, \& Barrouillet, 1997; Fayol, Abdi, \& Gombert, 1987; Thevenot, 2010; Thevenot, Devidal, Barrouillet, \& Fayol, 2007). In our case, Experiment 4's results showed that participants use different solving algorithms to solve isomorphic problems, depending on the type of quantities these problems mention. In the seminal description of the schema theory, Kintsch and Greeno (1985) indicate that the activation of a schema to solve a problem is done based on the propositional structure of the problem statement. In other words, the relevant entities are the numerical values and the relations explicitly described in the problems. The model proposed by Kintsch and Greeno does not explicitly raise the possibility that the world knowledge evoked by the elements mentioned in a problem may influence solvers' representation of the problems, nor their choice of a solving algorithm. Thus, those results could not have been predicted by the schema theory.

Since 1990, an alternative approach has emerged from Reusser's critical observations on the schema theory: the Situation-Problem-Solver, a model introducing the idea that the text of the problem statement is first translated into a situation model of the situation, before being "mathematized" into a problem model and finally translated into a solving algorithm (Reusser, 1990; Staub \& Reusser, 1995). Yet, it has been argued that by relying on the notion of situation model, the Situation-Problem-Solver approach neglects some interpretative effects at play in mathematical word problem solving. Indeed, this model does not directly include the idea that different models of a given problem statement can be built, depending on the solver's viewpoint (Gros, Thibaut, \& Sander, 2020). More specifically, this approach struggles to account for the idea that the initial problem representation constructed by the solvers may vary depending on the state of their general, non-mathematical knowledge about the elements present in the problems. Moreover, the notion that participants may overcome their initial encoding of a problem and construct a new representation also falls outside of the Situation-Problem-Solver's scope. However, in Experiments 5 and 6, participants were shown to engage in a semantic recoding process, akin to rerepresentation (Vicente et al., 2007), when their initial representation of the problem led to a dead end.

Thus, the schema theory and the Situation-Problem-Solver model both struggle to account for the results of our six experiments. On the other hand, those results are well within the scope of the new Semantic Congruence (SECO) model, which provides a conceptual framework for arithmetic word problem solving (Gros et al., 2020). The SECO model is meant to account for the influence of world semantics (the non-mathematical world knowledge one has about the entities described in a problem statement) on the initial encoding of a word problem into a specific interpreted structure (see Fig. 9). It proposes that when reading a problem statement, most solvers 
resort to their world semantics as well as to their mathematical semantics (the solver's mathematical knowledge applicable to the problem statement) to construct a representation of the described situation (an interpreted structure), which can then be translated into specific solving algorithms. When no relevant solving algorithm can be specified from the interpreted structure, then solvers may attempt to engage in semantic recoding by using mathematical knowledge to construct a new representation of the problem at hand. When performed successfully, this semantic recoding makes it possible to use different solving algorithms compatible with the new constructed representation (Gros et al., 2020).

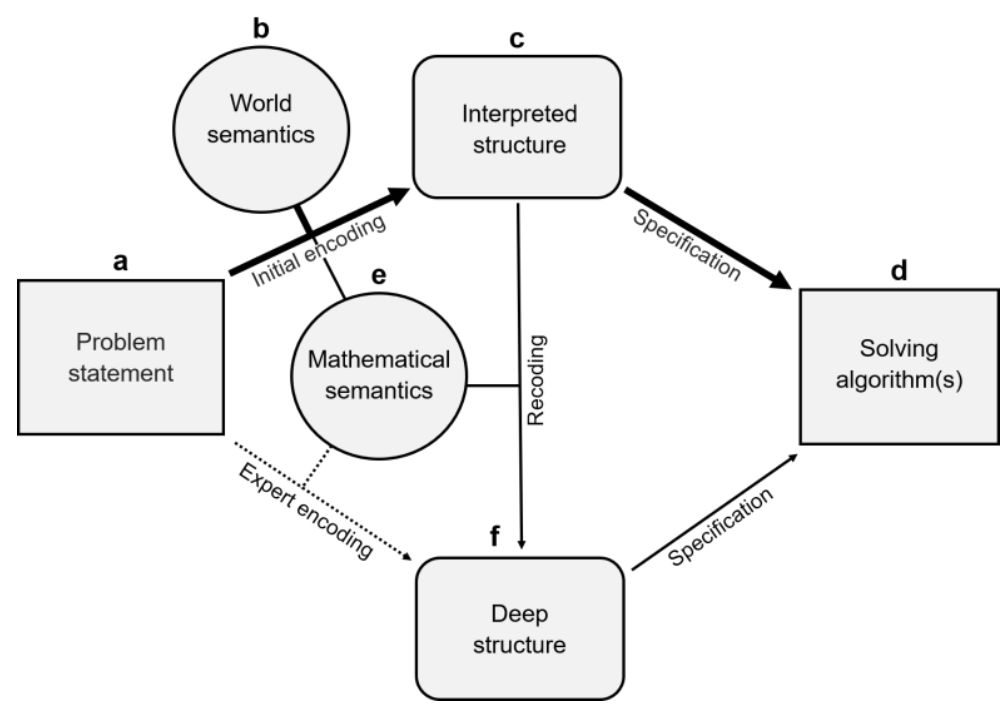

Fig. 9: Structure of the SECO model. Reprinted from "Semantic congruence in arithmetic: A new model for word problem solving," by H. Gros, J.-P. Thibaut, \& E. Sander, 2020, Educational Psychologist, 55, pp. 00461520.

For instance, in the case of the problems used in this study, SECO would predict that a cardinal problem featuring marbles will activate the solver's world knowledge about marbles (e.g. the fact that marbles are unordered entities that are usually grouped in no specific order) which will lead to the encoding of a cardinal interpreted structure, which then leads to the use of a 3-step algorithm (see Experiment 4). However, when a numerical value is removed from the problem and the 3-step algorithm cannot be used (see Experiments 5 and 6), participants need to discard the irrelevant surface features of the problem and engage in a semantic recoding process focused on the problem's mathematical properties (the notion that the difference between two sets sharing a common part is equal to the difference between their disjoint parts). If performed successfully, this recoding makes it possible for the participants to understand the relevance of the 1-step algorithm to solve cardinal problems. SECO suggests that engaging in such a recoding is cognitively costly, which explains the increased errors and reaction times on cardinal problems in these experiments. The results reported in this study thus support the relevance of the SECO model to account for arithmetic word problem solving, compared to previous 
approaches in the field.

\section{Potential confounds and interpretation}

The idea behind this study was to elicit either a cardinal or an ordinal encoding of problems sharing the same mathematical structure. Instead of resorting to digit ordering or magnitude comparison tasks to evaluate cardinal and ordinal understanding separately, we intended to show the relevance of these two dimensions within complex situations worded using natural language. The problems we created were thus meant to be identical in all respects except from their use of cardinal versus ordinal quantities, to avoid potential confounds such as a "garden path" effect in which participants are led to make an erroneous deduction about a sentence or a problem's goal (Johnson, Moen, \& Thompson, 1988; Tomasello \& Herron, 1988). In our case, leading the participants "down the garden path" would have meant changing the problem statements' structure to lead participants to calculate irrelevant values in some versions of the problems but not in others. On the contrary, we were especially careful to present the same pieces of information regarding the mathematical structure of the problems in the same order and within the same sentences across problems. For instance, the second sentence of the problems always introduced the Part 2 of the problems, both in cardinal problems (e.g. "He also has blue marbles") and in ordinal problems (e.g. "He climbs on a table"). Thus, the fact that participants decided to calculate the value of Part 2 on cardinal problems but not on ordinal problems cannot be attributed to the order in which the different parts and wholes were mentioned: instead, we believe that the explanation can only be found in the semantic difference introduced between the problem statements. Besides, Experiments 5 and 6 demonstrate that even when it is impossible to calculate the intermediate value Part 2, participants continue to use different strategies on cardinal and on ordinal problems. This strengthens the claim that participants' struggle on cardinal problems is not due to a treacherous garden path leading them to calculate irrelevant intermediate values along the way, but to a deeper issue of problem encoding.

Similarly, we believe that the distinction between ordinal quantities and cardinal quantities is not a matter of how continuous they are, but rather of how likely they are to emphasize, respectively, the order between the numerical values or their individual magnitudes. In fact, quantities qualifying as "discrete" were featured both in cardinal problems ("collections" problems) and in ordinal problems ("number of floors" problems) and quantities usually qualifying as "continuous" were used both in cardinal problems ("prices" and "weights" problems) and in ordinal problems ("durations" and "heights" problems). Thus, no matter the relative continuity or discreteness of the numerical dimension used, the main factor accounting for the differences observed in all 6 experiments remained the cardinal-ordinal distinction. 
Finally, it should be noted that in Experiments 1 to 3, the main difference between the problems we used was whether they featured cardinal or ordinal quantities. Thus, the saliency of the distinction between cardinal and ordinal problems may have been slightly inflated by the explicit instructions to directly classify or compare the problems. However, the problems also varied on other aspects (e.g. some cardinal problems featured weights while other featured prices) that participants did not necessarily pick up on, which means that the cardinal/ordinal distinction was not the only distinction that could have driven participants' answers, but rather the most salient one. Additionally, Experiments 4 to 6 did not include any classification nor comparison tasks, and the results on these experiments demonstrated that participants' encoding of the problems differed between cardinal and ordinal problems, even when no prior indication was given regarding the existence of different problem categories. In other words, the distinction between cardinal and ordinal problems remained significant across experiments, regardless of whether the instructions primed participants for the existence of different problem categories.

\section{Educational perspectives}

The range of content effects we have put forward in this paper showcase the fact that even for problems involving only elementary arithmetic operations, supposedly acquired in primary school, humans hardly manage to completely disregard context, and their performances in problem solving suffer from this shortcoming. However, the influence of world knowledge on mathematical word problem solving is not surprising considering that, as suggested by the kind world hypothesis, it leads to the making of inferences which are correct most of the time, since the surface features of situations are highly correlated with deeper principles (Bassok et al., 2008; Blessing \& Ross, 1996; Gentner \& Medina, 1998; Goldstone, 1994; Trench \& Minervino, 2015). Consequently, people tend to rely on those superficial cues which help them find the solution. Difficulties arise when the world knowledge evoked by a problem and its deep structure are semantically incongruent. Individuals are then bound to struggle, and sometimes even fail. This is especially problematic since mathematics education does not usually control for content effects (Bassok et al., 1998; Lee, DeWolf, Bassok, \& Holyoak, 2016), which is partly due to mathematics being primarily considered the realm of abstraction (Davis, Hersh, \& Marchisotto, 2011; Russell, 1903).

Although arithmetic word problems are a central part of mathematics education and teachers are usually encouraged to provide real-world examples to illustrate the notions being taught (e.g. Richland, Stigler, \& Holyoak, 2012; Rivet \& Krajcik, 2008), the use of concrete examples to teach new notions has also been shown to have a detrimental effect on transfer (Day, Motz, \& Goldstone, 2015; Goldstone \& Sakamoto, 2003; Son \& Goldstone, 2009). Just as concreteness fading is proposed as a tool to improve transfer by resorting to increasingly 
abstract examples (Fyfe, McNeil, Son, \& Goldstone, 2014), it may be a promising route to develop a semantic congruence fading process using increasingly incongruent examples. In the case of the problems used in the current study, starting with teaching the 1-step algorithm on ordinal problems, then moving to hybrid problems and then to concrete problems may be a way to help learners acquire a better understanding of this algorithm, and consequently learn to use it in any situation, regardless of the semantics conveyed by the problem statement. We believe that building on semantic congruence to develop such training programs may help learners develop a richer understanding of cardinality and ordinality as well as a more flexible approach to the encoding of numerical situations in general.

\section{Author Note}

Data and materials for all experiments are available online at https://osf.io/kz6gh/?view_only=2f3fb6b910844e238ae58fef3c61168a. This research was supported by grants from the Regional Council of Burgundy, Paris Feder Grants (20159201AAO050S02982 \& 20169201AAO050S01845), the Experimental Fund for the Youth and French Ministry of Education (HAP10CRE-EXPE-S1), and from the French Ministry of Education and Future Investment Plan (CS-032-15-836ARITHM-0). We thank Jay Boussier and Katarina Gvozdic for helpful comments on an earlier draft of the manuscript. Parts of this work were presented at the $38^{\text {th }}$ Annual meeting of the Cognitive Science Society (Experiments 5 and 6). Correspondence concerning this article should be addressed to Hippolyte Gros, IDEA Lab, University of Geneva, Geneva, 1205, Switzerland. E-mail: hippolyte.gros@unige.ch. 


\section{References}

Bassok, M. (2001). Semantic alignments in mathematical word problems. The analogical mind: Perspectives from cognitive science, 401-433.

Bassok, M., Chase, V. M., \& Martin, S. A. (1998). Adding apples and oranges: Alignment of semantic and formal knowledge. Cognitive Psychology, 35, 99-134.

Bassok, M., Pedigo, S. F., \& Oskarsson, A. (2008). Priming addition facts with semantic relations. Journal of Experimental Psychology: Learning, Memory, and Cognition, 34(2), 343-352.

Bassok, M., Wu, L. L., \& Olseth, K. L. (1995). Judging a book by its cover: Interpretative effects of content on problem-solving transfer. Memory and Cognition, 23, 354-367.

Bermejo, V. (1996). Cardinality development and counting. Developmental Psychology, 32(2), 263-268.

Blessing, S. B., \& Ross, B. H. (1996). Content effects in problem categorization and problem solving. Journal of Experimental Psychology: Learning, Memory, and Cognition, 22(3), 792.

Bonato, M., Zorzi, M., \& Umiltà, C. (2012). When time is space: evidence for a mental time line. Neuroscience \& Biobehavioral Reviews, 36(10), 2257-2273.

Boroditsky, L. (2011). How language shapes thought. Scientific American, 304(2), 62-65.

Carpenter, T. P., \& Moser, J. M. (1982). The development of addition and subtraction problem-solving skills. In T. P. Carpenter, J. M. Moser \& T. A. Romberg (Eds.), Addition and subtraction: A cognitive perspective, (pp. 9-24). Hillsdale, NJ: Erlbaum.

Casasanto, D. (2008). Who's afraid of the big bad Whorf? Crosslinguistic differences in temporal language and thought. Language learning, 58, 63-79.

Chen, Z., Xu, M., Shang, D., Peng, G., \& Luo, B. (2014). Distinct representations of symbolic ordinality and quantity: evidence from neuropsychological investigations in a Chinese patient with Gerstmann's syndrome. Brain and cognition, 88, 14-20.

Chi, M. T. H., Feltovich, P. J., \& Glaser, R. (1981). Categorization and representation of physics problems by experts and novices. Cognitive Science, 5, 121-152.

Clément, E., \& Richard, J.-F. (1997). Knowledge of domain effects in problem representation: The case of Tower of Hanoi isomorphs. Thinking \& Reasoning, 3(2), 133-157.

Colomé, À., \& Noël, M. P. (2012). One first? Acquisition of the cardinal and ordinal uses of numbers in preschoolers. Journal of Experimental Child Psychology, 113(2), 233-247.

Condry, K. F., \& Spelke, E. S. (2008). The development of language and abstract concepts: The case of 
natural number. Journal of Experimental Psychology: General, 137(1), 22-38.

Cummins, D. D., Kintsch, W., Reusser, K., \& Weimer, R. (1988). The role of understanding in solving word problems. Cognitive psychology, 20(4), 405-438.

Dantzig, T. (1945). Number the language of science. A critical survey written for the cultured nonmathematician. New York, NY: The Macmillan Company.

Daroczy, G., Wolska, M., Meurers, W. D., \& Nuerk, H. C. (2015). Word problems: a review of linguistic and numerical factors contributing to their difficulty. Frontiers in psychology, 6, 348.

Davis, P., Hersh, R., \& Marchisotto, E. A. (2011). The mathematical experience. Springer Science \& Business Media.

Day, S. B., Motz, B. A., \& Goldstone, R. L. (2015). The cognitive costs of context: The effects of concreteness and immersiveness in instructional examples. Frontiers in Psychology, 6, 1876.

De Corte, E., Verschaffel, L., \& De Win, L. (1985). Influence of rewording verbal problems on children's problem representations and solution. Journal of Educational Psychology, 77, 460-470.

Delazer, M., \& Butterworth, B. (1997). A dissociation of number meanings. Cognitive Neuropsychology, 14(4), 613-636.

Devidal, M., Fayol, M., \& Barrouillet, P. (1997). Stratégies de lecture et résolution de problèmes arithmétiques. L'Année Psychologique, 97(1), 9-31.

Droit-Volet, S., \& Coull, J. (2015). The developmental emergence of the mental time-line: spatial and numerical distortion of time judgement. PloS one, 10(7), e0130465.

Fayol, M., Abdi, H., \& Gombert, J. E. (1987). Arithmetic problems formulation and working memory load. Cognition and Instruction, 4(3), 187-202.

Fischbein, H. (1987). Intuition in science and mathematics: An educational approach. (Vol. 5). Springer Science \& Business Media.

Fischer, F. E., \& Beckey, R. D. (1990). Beginning kindergarteners' perception of number. Perceptual and Motor Skills, 70(2), 419-425.

Frege, G. (1980). The Foundations of Arithmetic [1884], transl. J. Austin. Evanston, IL: Northwestern University Press.

Fuhrman, O., \& Boroditsky, L. (2010). Cross-cultural differences in mental representations of time: Evidence from an implicit nonlinguistic task. Cognitive Science, 34(8), 1430-1451.

Fuson, K. C. (1988). Children's counting and concepts of number. New York: Springer-Verlag. 
Fyfe, E. R., McNeil, N. M., Son, J. Y., \& Goldstone, R. L. (2014). Concreteness fading in mathematics and science instruction: A systematic review. Educational Psychology Review, 26(1), 9-25.

Gamo, S., Sander, E., \& Richard, J.-F. (2010). Transfer of strategy use by semantic recoding in arithmetic problem solving. Learning and Instruction, 20, 400-410.

Gelman, R., \& Gallistel, C. R. (1978). The child's concept of number. Cambridge, MA: Harvard University Press.

Gentner, D., \& Medina, J. (1998). Similarity and the development of rules. Cognition, 65(2-3), 263-297.

Goffin, C., \& Ansari, D. (2016). Beyond magnitude: Judging ordinality of symbolic number is unrelated to magnitude comparison and independently relates to individual differences in arithmetic. Cognition, 150, 68-76.

Goldstone, R. L. (1994). The role of similarity in categorization: Providing a groundwork. Cognition, 52(2), 125-157.

Goldstone, R. L., \& Sakamoto, Y. (2003). The transfer of abstract principles governing complex adaptive systems. Cognitive psychology, 46(4), 414-466.

Gros, H., Sander, E., \& Thibaut, J. -P. (2019). When masters of abstraction run into a concrete wall: Experts failing arithmetic word problems. Psychonomic bulletin \& review, 26(5), 1738-1746.

Gros, H., Thibaut, J. -P., \& Sander, E. (2020) Semantic Congruence in Arithmetic: A New Model for Word Problem Solving, Educational Psychologist, 55 (in press).

Hofstadter, D. R., \& Sander, E. (2013). Surfaces and essences: Analogy as the fuel and fire of thinking. New York: Basic Books.

Hood, B. M. (1998). Gravity does rule for falling events. Developmental Science, 1(1), 59-63.

Hood, B. M., Santos, L., \& Fieselman, S. (2000). Two-year-olds' naïve predictions for horizontal trajectories. Developmental Science, 3(3), 328-332.

Hudson, T. (1983). Correspondences and numerical differences between disjoint sets. Child Development, Vol. 54, No. 1, 84-90.

Izard, V., Streri, A., \& Spelke, E. S. (2014). Toward exact number: Young children use one-to-one correspondence to measure set identity but not numerical equality. Cognitive Psychology, 72, 27-53.

Johnson, P. E., Moen, J. B., \& Thompson, W. B. (1988) Garden Path Errors in Diagnostic Reasoning. In: Bolc L., Coombs M.J. (eds) Expert System Applications. Symbolic Computation (Artificial Intelligence). Springer, Berlin, Heidelberg. 
Kim, I. K., \& Spelke, E. S. (1992). Infants' sensitivity to effects of gravity on visible object motion. Journal of Experimental Psychology: Human Perception and Performance, 18(2), 385.

Kintsch, W. (1988). The role of knowledge in discourse comprehension: A construction-integration model. Psychological review, 95(2), 163.

Kintsch, W., \& Greeno, J. G. (1985). Understanding and solving word arithmetic problems. Psychological review, 92(1), 109.

Lakoff, G., \& Núñez, R. E. (2000). Where mathematics comes from: how the embodied mind brings mathematics into being (Nachdr.). New York, NY: Basic Books.

Le Corre, M., \& Carey, S. (2007). One, two, three, four, nothing more: An investigation of the conceptual sources of the verbal counting principles. Cognition, 105(2), 395-438.

Lee, H. S., DeWolf, M., Bassok, M., \& Holyoak, K. J. (2016). Conceptual and procedural distinctions between fractions and decimals: A cross-national comparison. Cognition, 147, 57-69.

Lyons, I. M., \& Beilock, S. L. (2013). Ordinality and the nature of symbolic numbers. Journal of Neuroscience, 33(43), 17052-17061.

Lyons, I. M., Vogel, S. E., \& Ansari, D. (2016). On the ordinality of numbers: A review of neural and behavioral studies. In Progress in brain research, 227, 187-221.

Matejko, A. A., Hutchison, J. E., \& Ansari, D. (2019). Developmental specialization of the left intraparietal sulcus for symbolic ordinal processing. Cortex, 114, 41-53.

Meyer, C., Barbiers, S., \& Weerman, F. (2016). Order and Ordinality: The Acquisition of Cardinals and Ordinals in Dutch. In Boston University Conference on Language Development (BUCLD) 40. MA: Cascadilla Press.

Miller, K., Major, S. M., Shu, H., \& Zhang, H. (2000). Ordinal knowledge: Number names and number concepts in Chinese and English. Canadian Journal of Experimental Psychology/Revue canadienne de psychologie expérimentale, 54(2), 129.

Miller, S. E., Marcovitch, S., Boseovski, J. J., \& Lewkowicz, D. J. (2015). Young children's ability to use ordinal labels in a spatial search task. Merrill-Palmer Quarterly, 61(3), 345-361.

Nesher, P., Greeno, J. G., \& Riley, M. S. (1982). The development of semantic categories for addition and subtraction. Educational Studies in Mathematics, 13(4), 373-394.

Northcote, M. T., \& Marshall, L. (2016). What mathematics calculations do adults do in their everyday lives?: Part 1 of a report on the Everyday Mathematics Project. Australian Primary Mathematics Classroom, 
$21(2), 8-17$.

Piaget, J. (1965). The child's conception of number. New York: W. W. Norton. (Original work published 1941).

Reusser, K. (1990). From text to situation to equation: Cognitive simulation of understanding and solving mathematical word problems. In H. Mandl, E. De Corte, N. Bennet \& H.F. Friedrich (Eds), Learning and Instruction, European Research in an International Context, Vol. II. New York: Pergamon Press.

Richland, L. E., Stigler, J. W., \& Holyoak, K. J. (2012). Teaching the conceptual structure of mathematics. Educational Psychologist, 47(3), 189-203.

Rivet, A. E., \& Krajcik, J. S. (2008). Contextualizing instruction: Leveraging students' prior knowledge and experiences to foster understanding of middle school science. Journal of Research in Science Teaching: The Official Journal of the National Association for Research in Science Teaching, 45(1), 79-100.

Rubinsten, O., Dana, S., Lavro, D., \& Berger, A. (2013). Processing ordinality and quantity: ERP evidence of separate mechanisms. Brain and Cognition, 82(2), 201-212.

Rumelhart, D. E. (1980). Schemata: The building blocks of cognition. In R.J. Spiro, B.C. Bruce, \& W.F. Brewer (Eds.), Theoretical issues in reading comprehension (pp. 33-58). Hillsdale, NJ: Erlbaum.

Russell, B. (1903). Principles of mathematics. Cambridge, UK: Cambridge University Press.

Russell, B. (1919). Introduction to mathematical philosophy. London: Allen \& Unwin.

Sarnecka, B. W., \& Carey, S. (2008). How counting represents number: What children must learn and when they learn it. Cognition, 108(3), 662-674.

Sarnecka, B. W., \& Lee, M. D. (2009). Levels of number knowledge during early childhood. Journal of Experimental Child Psychology, 103(3), 325-337.

Sasanguie, D., Lyons, I. M., De Smedt, B., \& Reynvoet, B. (2017). Unpacking symbolic number comparison and its relation with arithmetic in adults. Cognition, 165, 26-38.

Schank, R. C. (1975). The role of memory in language processing. In: C. N. Cofer (ed), The Structure of Human Memory. San Francisco. (1975): p. 162-189

Schank, R. C., \& Abelson, R. P. (1977). Scripts, plans and understanding: An inquiry into human knowledge structures. New York: Hillsdale.

Schoenfeld, A. (2009). Learning to think mathematically: Problem solving, metacognition, and sense-making in mathematics. Colección Digital Eudoxus, (7). 
Simon, T. J. (1997). Reconceptualizing the origins of number knowledge: A "non-numerical" account. Cognitive Development, 12(3), 349-372.

Son, J. Y., \& Goldstone, R. L. (2009). Contextualization in perspective. Cognition and Instruction, 27(1), 5189.

Squire, S., \& Bryant, P. (2002). From sharing to dividing: Young children's understanding of division. Developmental Science, 5(4), 452-466.

Stacey, K. (2005). The place of problem solving in contemporary mathematics curriculum documents. The Journal of Mathematical Behavior, 24(3-4), 341-350.

Staub, F. C., \& Reusser, K. (1995). The role of presentational structures in understanding and solving mathematical word problems. In C. A. Weaver, S. Mannes, \& C. R. Fletcher (Eds.), Discourse comprehension (pp. 285-305). Hillsdale, NJ: Lawrence Erlbaum Associates.

Suppes, P. (1972). Axiomatic set theory. New York, NY: Dover Publications.

Suzuki, R., \& Shimodaira, H. (2006). Pvclust: An R package for assessing the uncertainty in hierarchical clustering. Bioinformatics, 22(12), 1540-1542.

Thevenot, C. (2010). Arithmetic word problem solving: Evidence for the construction of a mental model. Acta psychologica, 133(1), 90-95.

Thevenot, C., Devidal, M., Barrouillet, P., \& Fayol, M. (2007). Why does placing the question before an arithmetic word problem improve performance? A situation model account. The Quarterly Journal of Experimental Psychology, 60(1), 43-56.

Thevenot, C., \& Oakhill, J. (2005). The strategic use of alternative representations in arithmetic word problem solving. Quarterly Journal of Experimental Psychology-A, 58(7), 1311-1323.

Tomasello, M., \& Herron, C. (1988). Down the garden path: Inducing and correcting overgeneralization errors in the foreign language classroom. Applied Psycholinguistics, 9(3), 237-246.

Trench, M., \& Minervino, R. A. (2015). The role of surface similarity in analogical retrieval: Bridging the gap between the naturalistic and the experimental traditions. Cognitive science, 39(6), 1292-1319.

Turconi, E., Jemel, B., Rossion, B., \& Seron, X. (2004). Electrophysiological evidence for differential processing of numerical quantity and order in humans. Cognitive Brain Research, 21(1), 22-38.

Turconi, E., \& Seron, X. (2002). Dissociation between order and quantity meaning in a patient with Gerstmann syndrome. Cortex: A Journal Devoted to the Study of the Nervous System and Behavior, $38(5), 911-914$. 
Vicente, S., Orrantia, J., \& Verschaffel, L. (2007). Influence of situational and conceptual rewording on word problem solving. British Journal of Educational Psychology, 77(4), 829-848.

Wasner, M., Moeller, K., Fischer, M. H., \& Nuerk, H. C. (2015). Related but not the same: Ordinality, cardinality and 1-to-1 correspondence in finger-based numerical representations. Journal of Cognitive Psychology, 27(4), 426-441.

Weger, U. W., \& Pratt, J. (2008). Time flies like an arrow: Space-time compatibility effects suggest the use of a mental timeline. Psychonomic Bulletin \& Review, 15(2), 426-430.

Wynn, K. (1992). Addition and subtraction by human infants. Nature, 358(6389), 749-750. 